\title{
The Luminosity Function of Quasars and
}

its Evolution: A Comparison of Optically

\section{Selected Quasars and Quasars Found}

in Radio Catalogs

by

V. Petrosian

SUIPR Report No. 502

January 1973

(NASA-CR-131111) THE LUMINOSITY FUNCTIO
OF QUASARS AND ITS EVOLUTION: A
COMPARISON OF OPTICALLY SELECTED QUASARS
AND QUASARS FOUND IN RADIO CATALOGS

(Stanford Univ.) 48 p HC $\$ 4.50$ CSCL 03A

National Science Foundation Grant GP-23840

8L $<$ gLLL

National Aeronautics and Space Administration

Grant NGR 05-020-510

INSTITUTE FOR PLASMA RESEARCH STANFORD UNIVERSITY, STANFORD, CALIFORNIA 
THE LUMINOSITY FUNCTION OF QUASARS AND ITS EVOLUTION:

A COMPARISON OF OPTICALLY SELECTED QUASARS AND QUASARS FOUND IN RADIO CATALOGS

\author{
by \\ V. Petrosian ${ }^{*}$
}
National Science Foundation
Grant GP-23840

National Aeronautics and Space Administration

Grant NGR 05-020-510

SUIPR Report No. 502

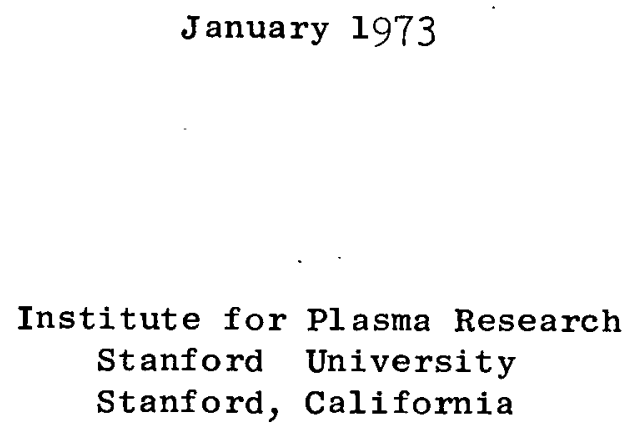

*Alfred P. Sloan Foundation Fellow I 
THE LUMINOSITY FUNCTION OF QUASARS AND ITS EVOLUTION:

A COMPARISON OF OPTICALLY SELECTED QUASARS AND

QUASARS FOUND IN RADIO CATALOGS

by

V. Petrosian ${ }^{*}$

Institute for Plasma Research

and Department of Applied Physics

Stanford University

Stanford, California

\section{ABSTRACT}

The luminosity function of quasars and its evolution are discussed, based on comparison of available data on optically selected quasars and quasars found in radio catalogs. It is assumed that the redshift of quasars is cosmological and the results are expressed in the framework of the $\Lambda=0$, $q_{0}=1$ cosmological model. The predictions of various density evolution laws are compared with observations of an optically selected sample of quasars and quasar samples from radio catalogs. The differences between the optical luminosity functions, the redshift distributions and the radio to optical luminosity ratios of optically selected quasars and radio quasars rule out Iuminosity functions where there is complete absence of correlation between radio and optical luminosities. These differences also imply that Schmidt's (1970) luminosity function, where there exists a statistical correlation between radio and optical luminosities, although may be correct for high redshift objects, disagrees with observation at low redshifts. These differences can be accounted for by postulating existence of two classes (I and II) of objects. The class I non-evolving objects dominate the optically selected samples at low redshifts, have low optical Iuminosities of about $10^{22} \mathrm{w} / \mathrm{Hz}($ at $2500 \stackrel{\circ}{\mathrm{A}}$ ) and are absent from radio samples because of their relative low radio to optical luminosity ratios. The class II objects are the quasars found in radio catalogs, show strong evolution, have optical luminosities of $10^{23}$ to $10^{24} \mathrm{w} / \mathrm{Hz}$ and dominate the optically selected samples at large redshifts. Possible relations between the two classes are also discussed, and tests for the validity of this picture are presented.

* Alfred P. Sloan Foundation Fellow<smiles>[C]1[C]CC1</smiles> 


\section{Introduction}

If the redshift of the quasar is cosmological then their distribution in space-time indicates that the universe is not in a steady state and that the quasars show strong evolution with cosmic time in conventional Friedmann models. This also means that the quasars cannot be used for the determination of the parameters (such as deceleration parameter, etc.) of the model for our universe. The subject of this paper is the evolution of the luminosity function of the quasars in the framework of a given cosmological model with the assumption that their redshift is cosmological. Unless otherwise stated, we shall assume the cosmological model with zero cosmological constant and with a deceleration parameter $q_{0}=1$. In Section II, we derive equations relating the luminosity function and its evolution to the observations of quasistellar radio sources (quasars observed first at radio frequencies and identified with optical objects subsequently) and optically selected quasars (or the so-called radio quiet quasars). The luminosity function and its evolution can be described by a function $\Psi(L, F, z)$ which gives the frequency distribution of the optical luminosity $L$ and radio luminosity $F$ of objects at different cosmological epochs or redshifts $z$. The results of comparison with observation are discussed in Section III where we compare various density evolution laws, and in Sections IV and $V$ where we discuss various possible luminosity functions and their extrapolation to low luminosities. In Section VI we present a modified $\mathrm{V} / \mathrm{V}_{\mathrm{m}}$ analysis for optically selected quasars. The results are summarized in Section VII. 


\section{Evolution of the Luminosity Function}

The luminosity function and its evolution can be represented by a single function $\Psi(L, F, z)$, which gives the frequency distribution of objects in the three dimensional space L, F, and $z$. [Following Schmidt (1968) L will refer to the luminosity at $2500 \AA$ (Schmidt's $F(2500)$ ) and $F$ will refer to the luminosity at $500 \mathrm{MHz}$ (Schmidt's $\mathrm{F}(500)$ ).] This functiongives an average statistical description of the variation of the optical and radio luminosities of the objects throughout their lifetime, their birth and extinction rates and the changes of these with cosmological epoch. It is hoped that the knowledge of the luminosity function $\Psi$ will give us some understanding of the physical processes which govern the behavior of these objects. The function $\Psi$ itself is deduced from observations of the redshift, optical intensity $I$, and radio flux density $S$ of the objects. In order to account for some selection effects, one needs this data for a sample of sources complete to a given optical intensity $I_{0}$ and radio flux density $\mathrm{S}_{0}$. Given this data one can calculate the optical and radio luminosities of each source in the framework of any cosmological model,

$$
\mathrm{F}=\mathrm{Sg}_{\mathrm{r}}(\mathrm{z}) \approx \mathrm{Sz}^{2}, \quad \mathrm{~L}=\mathrm{Ig}_{\mathrm{o}}(\mathrm{z}) \approx \mathrm{Iz}^{2}
$$

* Note that because we are interested in luminosities at a given frequency the functions $g_{r}$ and $g_{o}$ will be different due to the difference in the optical and radio spectra of the sources. If $S \propto v^{-\alpha_{r}}, \quad$ I $\propto v^{-\alpha_{0}}$, then for our cosmological model

$$
g_{x}(z)=4 \pi\left(\frac{c}{H_{o}}\right)^{2} z^{2}(1+z)^{1-\alpha_{x}} .
$$

Because $\left\langle\alpha_{\mathbf{r}}\right\rangle \sim\left\langle\alpha_{\mathrm{o}}\right\rangle \sim 0.7$, the $\mathrm{K}$-correction term $(1+\mathrm{z})^{1-\alpha_{\mathrm{x}}}$ is small and the same at radio and optical frequencies. We shall neglect this term in our discussion and set $4 \pi\left(\mathrm{c} / \mathrm{H}_{\mathrm{o}}\right)^{2}=1$ as indicated by equation (1). However, the numerical results are obtained including the K-correction terms and with $\left(\mathrm{c} / \mathrm{H}_{\mathrm{o}}\right)=3000 \mathrm{Mpc}$. Note also that $\mathrm{I}_{\mathrm{o}}$ is same as $F(2500)$ of Schmidt (1968). 
Then from $\mathrm{N}_{\mathrm{I}_{\mathrm{O}}}, \mathrm{S}_{\mathrm{O}}(\mathrm{F}, \mathrm{L})$, the observed number of sources in the sample with optical and radio luminosities $L$ to $L+d L$ and $F$ to $F+d F$ we have

$$
\int_{0}^{V_{m}} \Psi(L, F, z) V^{\prime} d z=N_{I_{0}, S_{o}}(F, L), \quad V_{m}=V\left(z_{m}\right)
$$

where $\mathrm{V}(\mathrm{z})$ is the co-moving volume up to a redshift $\mathrm{z}$ [for our model $\mathrm{V}^{\prime} \equiv$ $\left.\frac{d V}{d z} \propto \frac{z^{2}}{(1+z) \sqrt[3]{1+2 z}}\right]$ and $z_{m}$ is the maximum redshift within which the objects of luminosities $L$ and $F$ could be observed given the limits $I_{0}, S_{0}$.

$$
\begin{gathered}
z_{m}=\sqrt{\left(\frac{F}{S_{0}}\right)}, \quad \frac{I}{S}>\frac{I_{0}}{S_{0}} \text { radio limited } \\
z_{m}=\sqrt{\left(\frac{L}{I_{0}}\right)}, \quad \frac{I}{S}<\frac{I_{0}}{S_{0}} \text { optically limited. }
\end{gathered}
$$

We can also write

$$
\Psi\left(L, F, z_{m}\right) \propto \frac{d_{I_{o}, S_{o}}(L, F)}{d z_{m}} \times\left(1+z_{m}\right) \sqrt[3]{1+2 z_{m}} / z_{m}^{2}
$$

so that by comparing the number of sources in samples limited to different $I_{0}, S_{0}$ we can calculate $\Psi$.

Unfortunately, with the limited data available at the present time one cannot deduce a complete description of the distribution in $L, F$ and $z$. For example, the 3CR sample of Schmidt (1967) and the 4C sample of Lynds and Wills (1971), each contain about 30 objects. Subdividing these samples into various luminosity classes and various limiting observed intensities (or maximum redshifts, $z_{m}{ }^{\prime} s$ ) will give only few objects in each subgroup and reduce the statistical significance of the results. Furthermore, since low luminosity objects can be seen only when they are nearby we cannot learn about the behavior of these objects at large redshifts. The high luminosity objects, on the other hand, are less numerous and we must sample a larger volume of space to observe them. The probability of finding 
these objects at small redshifts is less than the probability of seeing them at large redshift so that one cannot learn about the behavior of $\Psi$ for the high luminosity objects at low redshifts. Because of these limitations it is necessary to compare some average integral property of the function $\Psi$ with observations. The one moment of $\Psi$ used in recent years for comparison with observation, which renders to simple interpretation in special cases, is the average value of $\mathrm{V} / \mathrm{V}_{\mathrm{m}}$ [suggested first by Kafka (1967) and applied to 3CR sources by Schmidt (1968) and by Lynds and Wills (1972) to $4 \mathrm{c}$ sources]. In the Appendix we show the expression for $\left\langle\mathrm{V} / \mathrm{v}_{\mathrm{m}}\right\rangle$ for quasistellar radio sources which have two limiting fluxes, radio and optical. However, the essential feature of this test can be seen more clearly if we consider a sample of sources with only one limiting flux. This will be the case for a sample of optically selected quasars where only the optical limit must be taken into account. If we integrate over all radio luminosities to obtain the optical luminosity function

$$
\Psi_{\text {opt }}(L, z)=\int_{0}^{\infty} \Psi(L, F, z) d F
$$

then the average value of $\mathrm{V} / \mathrm{V}_{\mathrm{m}}$ is given by

$$
\langle x\rangle \equiv\left\langle\mathrm{v} / \mathrm{v}_{\mathrm{m}}\right\rangle=\frac{\int_{0}^{\infty} \mathrm{dLV} \mathrm{m}^{-1} \int_{0}^{\mathrm{V}_{\mathrm{m}}} \mathrm{V} \Psi_{o p t}(\mathrm{~L}, \mathrm{z}) \mathrm{V}^{\prime} \mathrm{dz}}{\int_{0}^{\infty} \mathrm{dL} \int_{0}^{V_{\mathrm{m}}} \Psi_{o p t}(L, z) \mathrm{V}^{\prime} \mathrm{dz}} .
$$

It is clear that if the luminosity function does not evolve ( $\Psi_{\text {opt }}(L, z)$ independent of $z$ ) then $\langle x\rangle=0.5$. In general, if the observed $\langle x\rangle=0.5$, evolution cannot be ruled out. But if $\langle x\rangle \neq 0.5$ then there must be some evolution of the luminosity function. In order to proceed further and determine the possible evolutionary laws we must make some simplifying assumption about the luminosity function. The various ways that the luminosity function can be written and the interpretation of these ways have 
been discussed elsewhere (Lynds and Petrosian 1972). We shall limit our discussion here to the simplest type of evolution, namely the case of pure density evolution where it is assumed that the shape (dependence on $L$ and $F$ ) of the luminosity function is epoch independent, so that one can write for the general case

$$
\Psi(L, F, z)=\Psi(L, F) \rho(z), \quad \int_{0}^{\infty} \int_{0}^{\infty} \Psi(L, F) d L d F=1,
$$

and for the case of one limiting flux (equation (6))

$$
\Psi_{\text {opt }}(L, z)=\Psi_{o p t}(L) \rho(z), \quad \int_{0}^{\infty} \Psi_{o p t}(L) d L=1 .
$$

With this assumption, expressions (3) and (5) for the luminosity function are simplified as

$$
\Psi(\mathrm{L}, \mathrm{F})=\mathrm{N}_{\mathrm{I}_{\mathrm{O}}, \mathrm{S}_{\mathrm{o}}}(\mathrm{F}, \mathrm{L}) /\left[\int_{0}^{\mathrm{V}_{\mathrm{m}}(\mathrm{L}, \mathrm{F})} \rho(\mathrm{z}) \mathrm{v}^{\prime} \mathrm{dz}\right],
$$

and for the case of one limiting flux

$$
\Psi_{\text {opt }}(L)=N_{I_{0}}(L) /\left[\int_{0}^{V_{m}} \rho(z) v^{\prime} d z\right] .
$$

where $\mathrm{N}_{I_{O}}(L)$ is the number of sources with luminosity $L$ to $L+d L$ in an optically selected sample of sources with $I>I_{0}$. Substitution of equations (9) and (11) into equation ( 7 ) gives

$$
\langle x\rangle=\frac{1}{N_{I_{0}}} \int_{0}^{\infty} N_{I_{0}}(L) x(L) d L=\frac{1}{N_{I_{0}}} \sum_{i=1}^{N_{I_{0}}} x\left(L_{i}\right) \text {, }
$$

where

$$
x(L)=\frac{\int_{0}^{z} v_{\rho}(z) v^{\prime} d z}{v_{m} \int_{0}^{z_{m}} \rho(z) v^{\prime} d z},
$$


$N_{I_{0}}=\int_{0}^{\infty} N_{I_{0}}(L) d L$ is the total number of sources in a sample complete to a flux density $I_{0}$, and $z_{m}=\sqrt{L / I_{0}}$.

From observed values of $x(L)$ and $\langle x\rangle$ and equations (12) and (13)

one can determine the law of the evolution of density $\rho(z)$. Then with the help of this law and equation (10) one can calculate the luminosity function. 
On Table 1 we summarize the observed values of $\left\langle\mathrm{V} / \mathrm{V}_{\mathrm{m}}\right\rangle$ for the $3 \mathrm{CR}$ sample (Schmidt 1968), the 4C sample of Lynds and Wills (1972) and a sample of optically selected objects (Lynds et al (1972)). (The $V / V_{m}$ values for the latter sample were obtained by a slightly different method. This method is described in Section VI.) The first number gives the value of $\langle x\rangle$ for the whole sample. The second and third numbers give the values of $\langle x\rangle$ for the high and low $z_{m}$ (or luminosity) objects, respectively. The number of sources, $N$, and the expected standard deviation $\sigma=\frac{1}{\sqrt{12 \mathrm{~N}}}$ are also given for each sample. No useful information can be obtained from further subdivision of the samples because of large values of the $\sigma^{\prime} s$.

It is clear that with such meager data one cannot give a unique description of the function $\rho(z)$. As a result a few single parameter functions have been considered. We shall consider here the following functional forms for $\rho(z)$.

$$
\begin{gathered}
\rho(z)=\rho_{0}(1+z)^{m} \\
\rho(z) \propto(V(z))^{\alpha} \\
\rho(z)=\rho_{0} \exp \left(\beta^{\top}(z)\right) \\
\rho(z)=\rho_{0} \exp \left\{\beta^{\prime} z /(1+z)\right\}
\end{gathered}
$$

where $\tau(z)$ is the look back time in units of Hubble age $\mathrm{H}_{0}^{-1}$. For the cosmological model under consideration here

$$
\tau(z)=2 \tan ^{-1} \sqrt{1+2 z}+\sqrt{1+2 z} /(1+z)-(1+\pi / 2)
$$

The redshift dependences of the various evolution laws are shown in Figure 1. Figure 2 shows the variation of $x\left(z_{m}\right)$ with $z_{m}$ (which is equivalent to the variation of $x(L)$ with $L)$ for the above density evolution laws 
(equation 13). An evolution law nearly identical to a power law was first suggested for quasars by Schmidt. From theoretical considerations a power law in $(1+z)$ appears reasonable on the grounds that most physical quantities (such as average mass density etc.) in the universe, which could possibly effect the density of objects vary with some power of $(1+z) \quad(s e e$, for example, Rees and Setti, 1968). However, this law clearly cannot be extended to large redshifts because it predicts more objects with redshift $>2.5$ than has been observed (Petrosian, 1970) and because it would result in an isotropic background intensity at meter wavelengths much larger than observed. Furthermore, as it was pointed out by Lynds and Wills (1971), for the $3 C R$ and $4 C$ samples the observed values of $\langle x\rangle$ for high and low luminosity objects are equal, while the expected values of $\langle x\rangle$ from a power law density evolution for samples with the same $z_{m}$ (or luminosity) distribution as the $3 C R$ and $4 C$ samples are different (cf. Table 1).

Although for each sample alone the differences between the expected and observed values of $\langle x\rangle$ are not statistically significant, the fact that both samples show similar behavior was considered significant by Lynds and Wills (1972) who suggested the density evolution law of equation (15) for which (as is evident from equation (13)) $x(L$ ) is independent of $L$ and

$$
\langle\mathrm{x}\rangle=\frac{\alpha+1}{\alpha+2}
$$

This is also true for the general luminosity function (see Appendix 1). The unsatisfactory features of this evolution law are: (i) it implies zero density of objects at the present epoch, and (ii) it is difficult to find a physical relation between the co-moving volume and the density of sources.

The objections to the above evolution laws could be overcome by slight modifications. For example, modifying equation (14) so that the exponent $m$ is a decreasing function of redshift will overcome the difficulties 
encountered with that evolution law. Similarly if equation (15) is modified to read $\rho(z)=a+b v^{\alpha}$, the present density of objects in the universe becomes finite.

Exponential evolution laws similar to equation (16) have been suggested on various occasions. This law was used first by Rowan-Robinson (1967), and later by Doroshkevich et al. (1970) in connection with the evolution of radio sources. In an earlier paper (1970) I have shown that an exponential law of equation (16b) fits the redshift distribution of quasars without requiring an additional cutoff at larger redshifts. As is evident from Figures (1) and (2), the exponential laws predict slow variation of $x(L)$ with L. Table 1 shows the expected values of $\langle x\rangle$ for the evolution law of equation (16a) which are in better agreement with the observed values than the power law. Lynden-Bell (1972) has arrived at essentially the same conclusion using a different analysis of the 3CR data. Thus, the exponential laws overcome the objections raised against the previous two laws. Furthermore, an exponential evolution law could come about if the processes of the physical evolution of the sources (i.e. variation of their luminosity with their proper time) are statistical in nature as in nuclear radioactivity. Such a model has been discussed by Bahcall (1972).

Let us now consider the optically selected sample of Lynds et al. (1972). As is evident from Table 1 , for this sample, the values of $\langle x\rangle$ for the high and low $z_{m}$ objects are different: This is in good agreement with the expected values from a power law density evolution but shows statistically significant deviation from the expected values of the exponential laws or the law of equation (15). This evidence clearly reduces the significance of the disagreement between the observed behavior of the $3 \mathrm{CR}$ and $4 \mathrm{C}$ samples and the expected behavior of the power law density evolution. However, if the $3 \mathrm{CR}$ and $4 \mathrm{C}$ data are taken as evidence for the exponential law of 
evolution (or that of equation (15)), then we must modify these laws at low redshifts. Since the value of $\langle x\rangle$ for the low $z_{m}$ objects in the optically selected sample is not significantly different from 0.5 we may modify equation (15) or (16) by adding a constant term on the right hand side. For example, if we modify equation (15) to read

$$
\rho(v)=\rho_{0}\left(1+\left[\frac{v(z)}{v\left(z_{o}\right)}\right]^{\alpha}\right)
$$

then from equation (13) we have

$$
x\left(z_{m}\right)=\frac{\alpha+1}{\alpha+2} \frac{1+\left(v_{o} / v_{m}\right)^{\alpha}(1+\alpha / 2)}{1+\left(v_{o} / v_{m}\right)^{\alpha}(1+\alpha)}= \begin{cases}\frac{1}{2}, v_{m} \ll v\left(z_{o}\right) \\ \frac{\alpha+1}{\alpha+2}, & v_{m} \gg v\left(z_{o}\right)\end{cases}
$$

which by proper choice of $z_{o}\left(z_{o} \approx 0.6\right)$ could give the observed difference between the values of $\langle x\rangle$ for high and low $z_{m}$ objects. Note that this also overcomes one of the objections raised against this evolution law earlier. Similarly equation (16a) can be modified as

$$
\rho(z)=\rho_{I}+\rho_{I I} e^{\beta T(z)}
$$

Note that these modifications imply the existence of two classes of quasars. One class (class I) which shows no evolution and consequently is observed primarily at low redshifts. A second class (class II) which at the present epoch are less numerous than the first class but which have evolved such that at earlier epochs they were more numerous. Note also that the ten out of the eleven objects with low values of $z_{m}$ in the optically selected sample have measured redshifts $z<0.4$ while the average redshift of the other 12 objects is about 1.4. As we shall see in section V there appears to be other indications of differences between high redshift $(z \sim 1$ to 2$)$ and low redshift $(z<0.6)$ objects. 
In summary, if we assume that quasars have undergone density evolution then the combined $\mathrm{V} / \mathrm{V}_{\mathrm{m}}$ analysis of $3 \mathrm{CR}$, $4 \mathrm{C}$ and optically selected quasar data shows that a power law density evolution, although not the best fitting law, nevertheless is acceptable within the statistical uncertainty of the data. On the other hand, if we accept the $3 \mathrm{CR}$ and $4 \mathrm{C}$ data as evidence for exponential type evolution, then the data on radio quiet objects indicates the existence of two classes of quasars. 
Assuming density evolution then equation (10) and (II) and the density evolution law obtained from the $\mathrm{V} / \mathrm{V}_{\mathrm{m}}$ analysis give the luminosity function. If the assumption of density evolution is correct the above luminosity function would be valid for all epochs, otherwise it only would be an average luminosity function for the range of the observed redshifts. For samples consisting of small number of sources these equations must be integrated over a considerable range of luminosities so that it includes contribution from a few objects. Thus in practice one calculates

$$
\Phi_{1}(L, F)=\int_{L}^{L \times \Delta} d L^{\prime} \int_{F}^{F \times \Delta} d F^{\prime} \Psi\left(L^{\prime}, F^{\prime}\right)=\sum_{i} \frac{1}{\int_{0}^{V_{m i} \rho(z) d V}},
$$

where the summation includes contribution from all objects with observed luminosities in the indicated ranges. This procedure has been applied to the 3CR and the 4C sample by Lynds and Wills (1972) who present their results (for $\Delta=2.5$ ) in tabular form and flot $\Phi_{1}(F, L)$ versus $F$ for various values of the optical luminosity and versus $L$ for various values of the radio luminosity. It appears from these plots that the radio luminosity function can be fitted to a power law with a power exponent which is approximately the same for all optical luminosities. The optical luminosity functions are flat (with a steep cutoff for $L \geqslant 10^{23.8} \mathrm{w} / \mathrm{Hz}$ ) and show larger dispersion in the power exponent. If the above exponents were indeed independent of radio and optical luminosities we could treat $L$ and $F$ as stochastically independent parameters and write

$$
\Psi(L, F)=\Psi_{\text {opt }}(L) \Psi_{\operatorname{rad}}(F)
$$

In order to reduce the scatter in the data we have plotted in Figure 3 , the cumulative luminosity function 


$$
\Phi(\mathrm{L}, \mathrm{F})=\int_{\mathrm{F}}^{\infty} \mathrm{dF^{ \prime }} \int_{L}^{\infty} \Psi\left(\mathrm{L}^{\prime}, \mathrm{F}^{\prime}\right) \mathrm{dL^{ \prime }}
$$

versus $F$ and $L$ for various values of $L$ and $F$ respectively (because of steepness of the luminosity function the cumulative luminosity function is nearly identical to the function $\Phi_{1}$ in equation 21). As can be seen from these graphs identical curves could be fitted to all these points (although the curves plotted are not the best fitting curves) implying that equation (16) is approximately valid. The curves shown in Figure (3) can be expressed analytically as

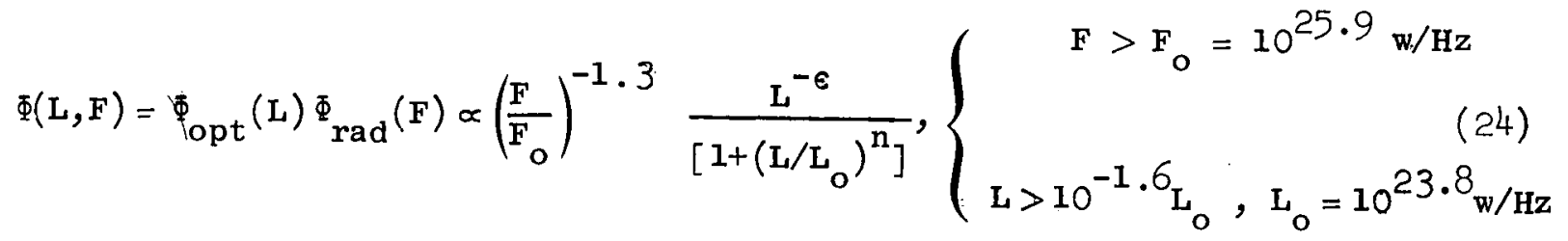

which approximately corresponds to a differential luminosity function of the form

$$
\Psi(L, F) \propto F^{-2 \cdot 3} L^{-1 * \epsilon} \frac{1}{\left[1+\left(L / L_{O}\right)^{n}\right.},\left\{\begin{array}{l}
F>F_{0} \\
L>10^{-1.6} L_{O}
\end{array}\right.
$$

The curves on Figure $(3 b)$ are for $\epsilon=0.3$. The data allow values of $\epsilon$ ranging from 0 to 0.5 . The term $\left[1+\left(L / L_{0}\right)^{n}\right]$ with $n \geq 1$ describes the apparent cutoff for $\mathrm{L} \geq 10^{24} \mathrm{w} / \mathrm{Hz}$. No such term seems to be necessary for high radio luminosities.

of course there are no compelling reasons to write the luminosity function as in equation (22). For a small sample of sources, it is possible to find other uncorrelated pairs of variables $X=f_{1}(L, F), Y=f_{2}(L, F)$, in which case one can repeat the above analysis (equation (21) onward) by changing $L$ and $F$ to $X$ and $Y$. For example, Schmidt (1970) has suggested that the radio and optical luminosities are statistically correlated so that one must write the luminosity function in terms of the ratio $R=F / L$ and $L$ 
in which case instead of equations (22) and (23) we have a new luminosity function

$$
\begin{gathered}
\Psi^{\prime}(\mathrm{L}, \mathrm{R})=\Psi_{\text {opt }}^{\prime}(\mathrm{L}) \Psi_{R}^{\prime}(\mathrm{R}), \\
\Phi^{\prime}(\mathrm{L}, \mathrm{R})=\int_{L}^{\infty} \mathrm{dL}^{\prime} \int_{\mathrm{R}}^{\infty} \mathrm{dR}^{\prime} \Psi^{\prime}\left(\mathrm{L}^{\prime}, \mathrm{R}^{\prime}\right)=\sum_{i} \frac{1}{\int_{0}^{V_{\mathrm{mi}}} \rho(\mathrm{V}) \mathrm{dV}} .
\end{gathered}
$$

As before, equation (27) expresses the new luminosity function in terms of the observed quantities, so that it can also be derived from Table 3 of Lynds and Wills (1972). The results of this derivation are shown on Figure 4 where $\Phi^{\prime}$ is plotted for various values of $R$ and $L$ versus $L$ and $R$ respectively. The two representations (equations 22 and 26) are related as

$$
\Psi^{\prime}(\mathbf{L}, \mathbf{R}) \mathrm{dLdR}=\Psi(\mathbf{L}, \mathbf{F}) \mathrm{dLdF},
$$

so that the new cumulative luminosity function is given by

$$
\Phi^{\prime}(\mathrm{L}, \mathrm{R}) \cong \mathrm{R}^{-1 \cdot 3} \int_{\mathrm{L}}^{\infty} \mathrm{dL} \mathrm{L}^{\prime} \frac{1}{\mathrm{~L}^{\prime 2 \cdot 3+\epsilon}\left[1+\left(\frac{\mathrm{L}^{\prime}}{\mathrm{L}_{\mathrm{o}}}\right)^{\mathrm{n}}\right]} .
$$

For a given $L, \Phi^{\prime} \propto R^{-1.3}$. Curves representing this relation are shown in Figure (4a). These curves agree with the observed points to the same accuracy as the curves in Figure (3a). Similarly, for a given $R, \Phi^{\prime}(R, L) \propto L^{-1.3-\epsilon}$ for $L \ll L_{0}$ (dashed lines in Figure (4b) plotted for $\epsilon=0.2$ ) and $\Phi^{\prime}(L, R) \propto$ $L^{-n-1.3-\epsilon}$ for $L \gg L_{0}$. These relations and equation (29) can be approximated as

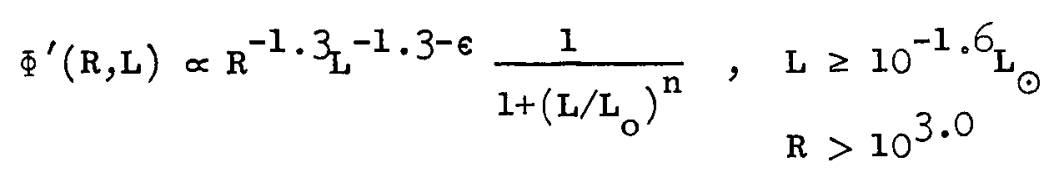

Lynden-Bell (1972) using a different method for the 3CR sample alone arrives at a similar luminosity function. 
Equations (24) and (30) are two different representations of the data on the quasistellar radio sources. They agree with observations to the same accuracy over the specified ranges of the luminosities. It is clear that these functions cannot be extended to very low luminosities. There must be a flattening of the optical luminosity functions (in both cases) for low values of $L$, and there must be a break in the power law for low values of $F$ (in equation (24)) and for low values of $R$ (in equation (30)). The position of this break is the distinguishing feature of these luminosity functions. If for all values of $L$ the break occurs at the same value of $F$ (or $R$ ) the correct representation of the luminosity function is given by equation (24) (or by equation (30)). As is evident from Figures (3) and (4) one cannot distinguish between the two luminosity functions based on quasistellar radio source data (3CR and 4C samples) alone. For example, these luminosity functions predict nearly identical redshift distributions for all samples with a ratio of $\frac{S_{0}}{I_{0}}$ comparable to that of the $3 \mathrm{CR}\left(\mathrm{S}_{\mathrm{o}} / \mathrm{I}_{\mathrm{o}}=9 \times 10^{4}\right)$ and the $4 \mathrm{C}\left(\mathrm{S}_{\mathrm{o}} / \mathrm{I}_{\mathrm{o}}=5.6 \times 10^{4}\right) \mathrm{sam}-$ ples. Only for samples with the ratio $s_{0} / I_{0} \ll<10^{4}$, in particular for any sample of optically selected quasars $\left(S_{0} / I_{0}=0\right)$, do the predictions of the two luminosity functions differ substantially. We compare the observations of optically selected quasars with the prediction of the two luminosity functions in the next section. 


\section{Extention of the Luminosity Function to Low Luminosities and Optically Selected Quasars}

In this section we compare the observed redshift distribution of quasars with the prediction of the two luminosity functions discussed in the previous section. For the case of stochastic independence of radio and optical luminosities (equation (22)) we adopt the cumulative luminosity function

$$
\begin{aligned}
& \Phi(L, F)=\Phi_{\text {opt }}(L)(F / F)^{-1.3}, \quad F>F_{o}=10^{25.9} \mathrm{w} / \mathrm{Hz} \\
& \Phi_{\text {opt }}(L)=\left\{\begin{array}{cl}
\left(L / L_{\min }\right)^{-\epsilon}\left[\frac{1+10^{-2 n}}{1+\left(L / L_{0}\right)^{n}}\right] & L>L_{\min }=10^{-L_{L}}=10^{21.8}{ }_{w / H z} \\
I & L<L_{\text {min }}, \quad n \geqslant 1
\end{array}\right.
\end{aligned}
$$

where we have not specified the form of the radio luminosity function for sources with luminosities (at $500 \mathrm{MHz}$ ) less than $10^{25.9} \mathrm{w} / \mathrm{Hz}$ and have assumed absence of sources with optical luminosities (at $2500 \AA$ ) less than $10^{21.8} \mathrm{w} / \mathrm{Hz}$. These numbers correspond to the lowest luminosities among al1 objects with known redshifts. The frequency distribution of redshifts of objects brighter than optical flux density $I_{0}$ and radio flux density $\mathrm{s}_{\mathrm{o}}$ is given by

$$
N(z)=V^{\prime} \rho(z) \cdot \Phi_{o p t}\left(I_{o} z^{2}\right) \Phi_{\operatorname{rad}}\left(S_{o} z^{2}\right)
$$

In Figure (5a)we present schematically the variation of this distribution with redshift. The top curve presents $v^{\prime} \rho(z)$ which is the redshift distribution of all observable sources $\left(I_{0}=S_{0}=0\right)$. For optically selected objects $\left(s_{0}=0, \Phi_{\text {rad }}=1\right)$ we obtain the lower solid curve, which begins to deviate from the upper curve at the redshift $z_{\text {opt }}=\left(L_{\min } / I_{0}\right)^{I / 2}$. At low 
values of $z$ the deviation is small and is a slowly varying function of $z$ because of the flatness of the optical luminosity function. The rapid cutoff at large $z\left[\left(z>\left(L_{0} / I_{0}\right)^{1 / 2}\right]\right.$, is due to the steepening of the Iuminosity function for $\mathrm{L} \geq \mathrm{L}_{\mathrm{O}}$.

For a sample of quasistellar radio sources $\left(s_{0}>0\right)$ we must also include the radio selection effects which is given by

$$
\Phi_{\operatorname{rad}}\left(S_{o} z^{2}\right)=\left(z / z_{r}\right)^{-2.6} \text { for } \quad z>z_{r} \equiv\left(F_{o} / S_{o}\right)^{1 / 2}
$$

if there are no sources with radio luminosity $F<F_{0}$. The dashed lines on Figure (5a) give the expected redshift distribution for two such samples, the top line for the case $S_{0} / I_{0}<F_{0} / L_{\min }$ and the lower line for $S_{0} / I_{0}>F_{o} / L_{\min }$ $\approx 10^{4}$. A's mentioned in the previous section, in practical cases $\mathrm{s}_{\mathrm{o}} / \mathrm{I}_{\mathrm{o}}>10^{4}$ so that the lower case is more commonly applicable.

As it has been pointed out earlier (Petrosian, 1970) these redshift distribution curves agree with the observed distribution of quasistellar radio sources ( $3 \mathrm{CR}$, and all known objects) but not with the redshift distribution of all known optically selected quasars, which has a double peaked distribution. As we shall see below this disagreement cannot be entirely accounted for by observational selection effects, so that this luminosity function cannot be applied to samples with $s_{0} / I_{0}<10^{4}$, unless it is modified, for example, as in equation (40) below.

Furthermore, analysis of optically'selected samples of quasars (Braccesi, 1972, Schmidt, 1970) show that the optical luminosity function is much steeper than that given by equation (31) (compare Figures $3 b$ and 6 ), which also speaks against the validity of this luminosity function for low radio luminosities. The observed steep luminosity function for the optically selected quasar would come about if the optical luminosity function becomes steeper for low values of radio luminosities. This means that $\epsilon$ in equation (31) is a function of the radio luminosity and it increases with decreasing $F$. There is, however 
no evidence for any systematic steepening of the optical luminosity function with decreasing $F$ for $F>F_{0}$. To determine whether rapid steepening of the optical luminosity function takes place for $F<F_{0}$ we must wait for data on samples with more objects and extending to lower values of $\mathrm{S}_{0}$.

A steep optical luminosity function would be observed if, as suggested by Schmidt (1970), the radio and optical luminosities were correlated so that the form of the optical luminosity function is independent of the value of $R=F / L$. Then we have equations (28) and (30) or

$$
\begin{aligned}
& \Phi^{\prime}(L, R)=\Phi_{o p t}^{\prime}(L) G(R)
\end{aligned}
$$

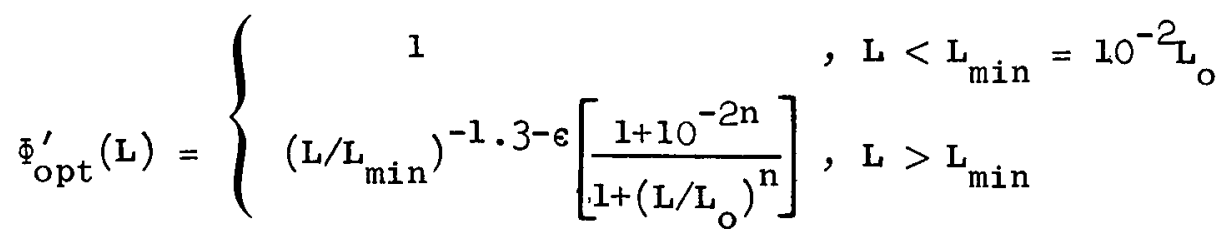

$$
\begin{aligned}
& G(R)=K\left(R / R_{0}\right)^{-1.3} \quad, R>R_{0}=10^{3.2} \\
& G\left(R_{\min }\right)=1
\end{aligned}
$$

where we have not specified the value of $R_{\min }$ or the form of the function $G(R)$ for $R_{0}>R>R_{\text {min }}$.

In this case the redshift distribution (for $z>z_{\text {opt }}$ ) of objects brighter than $I_{0}$ and $S_{0}$ is:

i) $0<\mathrm{S}_{\mathrm{O}}<\mathrm{I}_{\mathrm{O}} \mathrm{R}_{\mathrm{min}}$

$$
N(z)=\rho(z) v^{\prime} \begin{cases}1, & z<z_{\text {opt }} \equiv\left(L_{\text {min }} / I_{o}\right)^{1 / 2} \\ \Phi_{\text {opt }}^{\prime}\left(I_{o} z^{2}\right), & z>z_{\text {opt }}\end{cases}
$$

ii) $S_{0}>I_{0} R_{\min }$

$$
N(z)=\rho(z) V^{\prime} \begin{cases}1 & , \quad z<z_{r} \equiv\left(L_{\min } R_{\min } / S_{0}\right)^{1 / 2} \\ \Phi_{o p t}^{\prime}\left(\frac{S_{o} z^{2}}{R_{\text {min }}}\right)[1+X(z)], & z>z_{r}\end{cases}
$$


where

$$
X(z)=\frac{1}{\Phi^{\prime}\left(\frac{S_{0} z^{2}}{R_{\text {min }}}\right)} \int_{I_{o} z^{2}}^{S_{0} z^{2} / R_{\text {min }}} \Psi_{o p t}^{\prime}(L) G\left(\frac{S_{o} z^{2}}{L}\right) d L
$$

is a function of order unity changing slowly from zero at $z=z_{\mathbf{r}}$ to a constant value for $z \geq z_{\text {opt }}$. As shown schematically in Figure (5b) the redshift distribution for the quasistellar radio sources (i.e. $s_{o} / I_{0}>R_{\min }$ ) expected from equation (34) is identical to that expected from equation (32). However, for $S_{0} / I_{0} \leq R_{\min }$ (i.e. for optically selected quasars) the redshift distribution of equation (35) is nearly identical to the redshift distribution of quasistellar radio sources, because in this case unlike in equation (3I) - the optical luminosity function $\Psi_{\text {opt }}^{\prime}(L)$ is as steep as the radio luminosity function $\Psi_{\text {rad }}(F)$. In fact, the luminosity function of equation (34) was proposed by Schmidt (1970) when he observed a rough agreement between the redshift distribution of a sample of 18 magnitude optically selected quasars and the 3CR quasars.* However, as we shall discuss below, data from other optically selected samples of quasars does not agree with some of the predictions of this luminosity function. In addition, this luminosity function encounters the following difficulty.

Comparison of observed and expected values of $R$ : If equation (34) is valid for all radio luminosities (or for all values of $R$ ), then for any sample of optically selected quasars the average value of the ratio of radio to optical intensities (or luminosities) is given by

$$
\left\langle\frac{S}{I}\right\rangle=\left\langle\frac{F}{L}\right\rangle=\langle R\rangle=\int_{R_{\min }}^{\infty} R G^{\prime}(R) d R,
$$

where $G^{\prime}(R)=-d G(R) / d R$. There has been various attempts of radio

\footnotetext{
* The independence of redshift distribution from the radio flux density limit may also be considered as evidence for non-cosmological nature of the redshifts.
} 
observations of optically selected quasars. The most recent attempt by Wardle and Miley (1971) indicates positive detection of radio emission (at $2695 \mathrm{MHz}$ ) only from one object. For all other objects of known redshift they failed to detect any radio emission greater than a few times $10^{-29} \mathrm{w} /\left(\mathrm{m}^{2} \mathrm{~Hz}\right)$. Using the upper limit for each source from these data and assuming an average radio spectral index $\alpha_{\mathbf{r}} \sim 0.7$ we find that

$$
\langle R\rangle_{\text {observed }} \leq 200
$$

The expected average value of $R$ from equation (34) will depend on the behavior of the function $G(R)$ for $R \leq R_{0}=10^{3.2}$. Schmidt (1970), from comparison of $3 C R$ and radio quiet quasars, gives $G\left(R=R_{0}\right)=K \approx 0.05$. It can be shown that the smallest expected value for $\langle R\rangle$ is obtained if $G(R) \propto \log R$ for $R<R_{0}$, so that the expected value of $\langle R\rangle$ for the luminosity function of equation (34) is

$$
\langle R\rangle_{\text {eq. }} 34>500
$$

Note, for example the extention of $G(R)$ suggested by Schmidt (1970) gives $\langle R\rangle \approx 10^{3}$. The sample of all known optically selected quasars includes many PHL objects, few of which are identified with $4 \mathrm{C}$ sources. Since the redshifts of all PHL objects are not known it is not clear how much the identified PHL objects contribute to the value of $\langle R\rangle$ in equation (38). On the other hand, all the objects in the sample of Lynds et al. (1972) were included in Wardle and Miley's observation program so that for the 22 objects in this sample

$$
\langle R\rangle_{\text {observed }}<120
$$

compared to the expected value of equation (38). The discrepancy between equation (39) (or equation (37)) and equation (38) may be due to the 
difficulty in the radio observations of these objects. Wardle and Miley indicate that few of the objects are confused with strong radio sources. If it just happens that these objects are themselves strong emitters, the above discrepancy could be resolved. In a recent paper, Schmidt (1972) refers to observations by Katgert et.al. (unpublished) who have detected radio emission at $1410 \mathrm{MHz}$ from 2, or possibly 3, objects at the level of 0.01 flux units. If this data is confirmed, the above difficulty with the luminosity function of equation (34) will be alleviated. Otherwise this equation must be abandoned, partially or completely as a description of low radio luminosity quasars.

There are various ways of extending the observed luminosity function of quasistellar radio sources to lower radio luminosities which could overcome such discrepancies. For example, if the observed redshift distribution of optically selected quasars and their steep luminosity function is due to the steepening of the optical luminosity function of equation (31) for $F<F$ o rather than due to the existence of a correlation between optical and radio luminosities, then the luminosity function is given by equation (31) for $F>F_{0}$ and the optical luminosity function by

$$
\Phi\left(\mathrm{L}, \mathrm{F}_{\min }\right)=\int_{\mathrm{L}}^{\infty} \mathrm{dL^{ \prime }} \int_{F_{\min }}^{\infty} \mathrm{d} F^{\prime} \Psi\left(\mathrm{L}^{\prime}, F^{\prime}\right) \propto \mathrm{L}^{-1 \cdot 3-\epsilon},
$$

where $F_{\text {min }}$ is the smallest radio luminosity. In this case the expected values of $\langle F / L\rangle$ for optically selected quasars is given by

$$
\left\langle\frac{F}{L}\right\rangle \approx \frac{F \min }{\left\langle I z^{2}\right\rangle}
$$

For $\mathrm{F}_{\min } \lesssim 10^{25.4} \mathrm{w} / \mathrm{Hz}$ this ratio would be in agreement with the low observed value in equation (39). 
On the other hand, if our earlier hypothesis that most of the low redshift objects belong to a different class is correct (see also the discussion below), then the above comparison should be made for objects with $z \geq .6 ; \quad$ i.e., if we assume that the luminosity function in equation (34) is valid only for the high redshift objects, then the value of $K$ in equation (34) is reduced by a factor of about 2 which is the ratio of number of all objects to number of objects with $z \lesssim .6 . \quad$ Furthermore, the large redshift objects are optically weaker (on the average by a factor of 2) than the low redshift objects. Inclusion of these two factors would eliminate the factor of 4 discrepancy between equations (38) and (39). Comparison with observed redshift distribution: A more serious difficulty with the types of luminosity functions we have been discussing is their failure to explain the presence of relatively large number of low redshift objects among optically selected quasars. The observational data showing the relative abundance of low redshift objects $(z \lesssim 0.6)$ in various samples of quasars are summarized in Table II. For the complete 3CR sample (Schmidt, 1968) and the $4 \mathrm{C}$ sample (Lynds and Wills, 1972) the fraction, f, of objects with $z \lesssim 0.6$ is less than $30 \%$ while for the optically selected sample of Lynds et al (1972), which is complete to an infrared magnitude $i=17.65$, 11 out of 22 objects have redshifts less than 0.6 . The average value of visual minus infrared magnitudes for the Lynds et al (1972) sample is $\langle v-i\rangle=0.65^{*}$ which implies a visual limit of $v \approx 18.3$. From Figure 2 of Braccesi et al (1970), we estimate the difference between ordinary visual magnitudes $m_{v}$ and their magnitude $v$ to be $m_{v}-v \approx 0.05$, so that the limiting visual magnitude $m_{v}$ for this sample is similar to that of the 3CR sample $\left(m_{v} \approx 18.4\right)$. There is, however, one selection effect which

For definition of $i$ and $v$ magnitudes, cf. Braccesi et al, 1970. 
could account for some of the difference between these samples. The average $v-i$ colors of the objects with $z<0.6$ in the Lynds et al sample is 0.2 magnitude larger than for objects with $z<0.6$. Whatever the cause of this, it will produce an over-representation of low. redshift objects by a factor of $10^{0.20 \times 0.75} \approx 1.4$, where 0.75 is the observed slope of the number magnitude counts (Sandage and Luyten, 1969; Braccesi and Formiggini, 1969). Or, if we exclude objects with $17.45<i<17.65$ from the 1 ist for $z<0.6$, the fraction $f$ for the optically selected sample is reduced to $9 / 20$ as shown in Table II. Other differences in techniques of selection of objects for these two samples are small to account for the difference between the values of the fraction $f$. As can be seen from Figure 5 or from examination of equations (35) and (32), a larger value of $f$ for optically selected quasars compared to radio quasars is contrary to the prediction of both luminosity functions. It clearly rules out the luminosity function of equation (31). The luminosity function of equation (34) predicts nearly identical redshift distribution for all samples of quasars limited to the same optical flux density $I_{0}$ independent of their radio flux density limits. In fact, contrary to observations, this luminosity function predicts. (see Figure $5 \mathrm{~b}$ ) fewer low redshift objects (i.e., smaller values of $f$ ) for an optically selected samples compared to a radio sample.

In order to test the significance of the differences between the observed and expected behavior of the fraction $f$, we have carried out $\chi^{2}$ analysis comparing the redshift distribution of the Lynds et al (1972) sample (after correction for the above mentioned selection effect) with the distribution of the $3 \mathrm{CR}$ and $4 \mathrm{C}$ samples. For the $4 \mathrm{C}$ sample we have included only objects

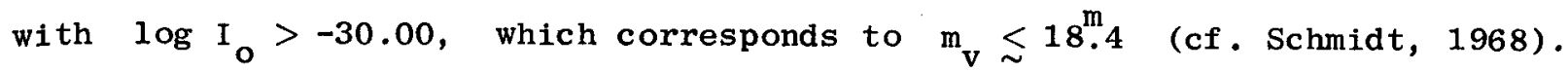
The difference between $4 C$ and $3 C R$ samples is not significant. The difference between the fractions given on Table II for the optically selected sample and 
the combined $3 \mathrm{CR}$ and $4 \mathrm{C}$ sample is larger but is not statistically significant. However, the dividing redshift $z=0.6$ was chosen to divide the complete Lynds et al (1972) sample into two equal parts. This sample shows more pronounced concentration of sources around $z \sim 0.3$, so that dividing the sample into two groups with $z<0.4$ and $z>0.4$ we find a probability of 0.06 that the difference between the optically selected sample and the combined $3 C R$ and $4 C$ samples is due to chance. Furthermore, if the samples are divided into three groups, each group containing approximately equal numbers of optically selected objects, we find a probability of less than 0.01 that the difference in the redshift distribution is due to chance. As a test for the validity of equation (34) these results indicate even smaller probabilities since as mentioned above this equation predicts fewer low redshift objects for an optically selected sample than for a radio sample.

Equation (34) predicts identical redshift distributions for samples of sources with a given optical intensity. Therefore, a better test of this equation will be the comparison between the redshift distribution of samples of sources within a narrow range of magnitudes. Since most of the sources in the observed samples have magnitudes around 18 magnitude, we consider now only 18 magnitude objects, i.e., sources with $-30.00<\log \mathrm{I}_{\mathrm{o}}<-29.6 \mathrm{w} /\left(\mathrm{Hz} \mathrm{m}^{2}\right)$. In this case we can also include in our test Schmidt's (1970) optically selected sample.

The individual redshifts of the objects in Schmidt's (1970) optically selected sample are not published. For the 18 magnitude objects in this sample the fraction $f=5 / 19$. There is one 18 magnitude object whose redshift is not known and there are three 17 magnitude ( $\left.\log I_{0}>-29.6\right)$ objects. Thus, as shown in Table II for the complete sample $f$ could be as large as 9/23, a number very similar to that of Lynds et al (1972) sample. On Table I we also list the fraction $f$ for the 18 magnitude objects in all 4 samples. 
For the Lynds et al sample we give the fraction $f$ obtained by including only objects with $16.45<i<17.45$ for $z<0.6$ and objects with $16.65<i<17.65$ for $z>0.6$. This accounts for the selection effect discussed above. $\chi^{2}$ analysis shows that the difference between the two optically selected samples is not statistically significant (the probability that this difference is due to chance is greater than 0.1). However, the probability that the difference between the combined optically selected sample and the combined samples from radio surveys $(3 \mathrm{CR}+4 \mathrm{C})$ is due to chance is less than 0.05 .

On Table II we also give the fraction $f$ for all quasars with known redshifts. The difference between $f$ for the optically selected quasars and quasars found in radio surveys is similar to the fractions discussed above. Since the sample of all known quasars is larger the probability that the difference in these samples (dividing the sample at $z \approx 0.4$ ) is due to chance is <.0005, a probability less than the probabilities obtained for the smaller but complete samples.

In summary we find that both luminosity fractions of equation (31) and (34) encounter difficulties when extended to low radio luminosities. Equation (31) disagrees with both the observed redshift distribution and the luminosity function of optically selected samples. Equation (34) agrees with the observed optical Iuminosity function but it disagrees with observed redshift distribution and predicts higher radio luminosities for quasars in optically selected samples than indicated by one set of observations (Wardle and Miley 1971).

We consider these evidences additional support for our earlier conjecture (Petrosian, 1972, see also the discussion at the end of Section III) about the presence of two classes of quasars. 
In this section we present calculations of $\left\langle V / V_{m}\right\rangle$ for the Lynds et al (1972) sample of quasars which is complete to an infrared magnitude of $i=$ 16.65. In previous calculations $\left\langle\mathrm{V} / \mathrm{V}_{\mathrm{m}}\right\rangle$ (Schmidt, 1968, Lynds and Wills, 1972) the sample was chosen to be complete to a given intensity at a rest frame wavelength of $2500 \AA$ after corrections for the effects of discrete lines. In this procedure few objects are eliminated from a sample that is complete to a given apparent magnitude. To avoid this loss of objects, a more complicated procedure could be used. We describe this procedure below.

Let us assume that the spectral luminosity $F(\nu)$ of an object can be described by a power law nonthermal source plus contribution from various lines of frequency $\nu_{i}$. Then

$$
F(\nu)=F\left(\nu_{0}\right)\left[\left(\frac{\nu}{\nu_{0}}\right)^{-\alpha_{0}}+\sum_{i}\left(\frac{\nu_{i}}{\nu_{0}}\right)^{-\alpha_{0}} w_{i} g\left(\nu-\nu_{i}\right)\right]
$$

where $g\left(\nu-\nu_{i}\right)$ describes the line shape $\left(\int_{-\infty}^{\infty} g(x) d x=1\right)$ and $w_{i}$ is the equivalent width of the line. If the source is at a redshift $z$ then the observed intensity at a frequency $\nu$ is

$$
I(\nu)=\frac{F\left(\nu_{0}\right)(1+z)}{z^{2}}\left\{\left(\frac{\nu(1+z)}{\nu_{0}}\right)^{-\alpha_{0}}+\sum\left(\frac{\nu_{i}}{\nu_{0}}\right)^{-\alpha_{0}} w_{i} g\left[\nu(1+z)-\nu_{i}\right]\right\}
$$

the observed equivalent width is $w_{i} /(1+z)$ and the observed magnitude $x$ for a filter response $s_{X}(\nu)$ is

$$
X=-2.5 \log \int_{0}^{\infty} I(\nu) s_{X}(\nu) d \nu .
$$

Since the line widths are in general much smaller than the filter response widths, we can approximate the line profile by a delta function and set $\nu_{0}$ equal to the center frequency of the filter to obtain 


$$
\begin{aligned}
& 10^{-0.4 X}=F(\nu) \frac{(1+z)^{1-\alpha}}{z^{2}} \int_{0}^{\infty}\left(\frac{\nu}{\nu}\right)^{-\alpha}{ }^{-} S_{x}(\nu) d \nu\left[1+\sum_{i} c_{i}(z)\right], \\
& c_{i}(z)=\frac{w_{i} s_{X}\left[\nu_{i} /(1+z)\right]}{(I+z)^{I-\alpha} \alpha_{0} \int_{0}^{\infty}\left(\frac{\nu}{\nu}\right)^{-\alpha_{0}} s_{X}(\nu) d \nu} \approx \frac{w_{i} s_{X}\left[\nu_{i} /(1+z)\right]}{(1+z) \int_{0}^{\infty} s_{X}(\nu) d \nu} .
\end{aligned}
$$

The last approximation is valid only if filter half power width $\Delta \nu_{X} \ll \nu_{0}$.

For a sample complete to an observed magnitude $x_{0}$ the maximum redshift $z_{m}$ up to which the objects would be observable is obtained from equations identical to equations (45) and (46) with $x$ replaced by $x_{0}$ and $z$ replaced by $z_{m}$. We can therefore write

$$
0.4\left(x_{0}-x\right)=2 \log \left(\frac{z_{m}}{z}\right)+\left(\alpha_{0}-1\right) \log \frac{1+z_{m}}{1+z}+\log \left[\frac{1+\sum_{i} c_{i}(z)}{1+\sum_{i} c_{i}\left(z_{m}\right)}\right] .
$$

In general only one strong line contributes to a given filter but different lines (if any) may contribute at $z$ and $z_{m}$. Furthermore, if the observed equivalent width $\frac{w_{i}}{1+z}$ is much smaller than the filter half power width $\Delta v_{X}$ the last term in equation (47) can be approximated as

$$
\log \left(1+c_{i}(z)\right) \approx \frac{1}{2 \cdot 3} \frac{w_{i} s_{X}\left(v_{i} /(1+z)\right)}{(1+z) \int_{0}^{\infty} s_{X}(v) d v}
$$

In order to calculate the values of $z_{m}$ we need to know the equivalent width of the strong lines and the response function $S_{X}(\nu)$. Since the sample under consideration is limited to infrared magnitude, i.e. $x=i$, we use the response function for this filter published by Braccesi et al. (1970). As this response function drops off rapidly for wavelengths $\lambda>7800 \stackrel{\circ}{A}$ the only strong lines effecting this filter (up to a redshift of 3) are $\mathrm{H} \alpha, \mathrm{HB}, \mathrm{MgII}$ and CIII at wavelengths $6562,486 \mathrm{I}, 2798$ and $1909 \stackrel{\circ}{\mathrm{A}}$ respectively. 
We assume that the equivalent widths are the same for all objects and we use equivalent widths (in wavelength units) of $550,163,47$, and $14 \AA$, respectively. These numbers were obtained by straight averaging of all rest frame equivalent widths, $w_{i}$, observed by Oke, Neugebauer, and Beklin (1970). Note that for calculating the value of $z_{m}$ by this method, a trial and error procedure must be used. Once $z_{m}$ is obtained we can then calculate the values of $\mathrm{V} / \mathrm{V}_{\mathrm{m}}$.

The results of these calculations are presented on Table III for two assumed values of the spectral index $\alpha_{0}$ and for the case where we have neglected the effect of the lines. As can be seen from this table there is a significant difference between the values of $\left\langle\mathrm{V} / \mathrm{v}_{\mathrm{m}}\right\rangle$ for high and low redshift (or luminosity) objects.

Columns 8 and 9 give the absolute luminosity of the sources calculated at two different wavelengths. The numbers in column 7 give the approximate infrared luminosities and are obtained from

$$
\log L_{\text {infrared }}=-\log z^{2} /(1+z)^{1-\alpha_{0}}-0.4(i+\Delta i)+C
$$

where $\Delta i$ corrects the infrared magnitude for the discrete lines, $C$ is an arbitrarily normalization factor and $\alpha_{0}=0.5$. The numbers in column 9 give the approximate values of logarithmic luminosities obtained from a relation similar to equation (49) with the infrared magnitude $i$ replaced by the ordinary visual magnitudes, $m_{v}$, with $\Delta i=0$, and with normalization $C$ chosen such that $m_{v}=18.4$ corresponds to $\log L=23 \mathrm{w} / \mathrm{Hz}$ for an object at $z=1$ (cf. Schmidt, 1968). These numbers do not exactly correspond to $\log \mathrm{L}$ (where $\mathrm{L}$ is the optical luminosity at $2500 \mathrm{~A}$ in units of $\mathrm{w} / \mathrm{Hz}$ ), their deviation from $\log \mathrm{L}$ is less than 0.1 , an accuracy which will suffice for the purpose of our discussion here. The value of the luminosities appear to divide the sample into two groups. The low redshift $(z \leq .4)$ objects all have $\log \mathrm{L} \sim 10^{22}$ except for $B 272$ which has narrow 
emission lines, Lynds et al. 1972, and will be neglected from the remainder of our discussion) while the large redshift objects have $\log \mathrm{L} \geqslant 22.8$ extending up to $\log \mathrm{L}=24.0$.

The last two columns on Table III give the inverse of the quantity

$$
v_{m}^{*} \equiv \int_{0}^{z_{m}} \rho(z) \frac{d V}{d z} d z
$$

which are needed for calculating the luminosity function (equation 11). The numbers in column 10 are obtained with $p(z)=(1+z)^{6}$ for the whole sample (cf. Table I) while the numbers in column 11 are calculated assuming $\rho(z)=$ const. for $z \leq 0.4$ and $\rho(z)=[v(z) / v(0.4)]^{\alpha}$ for $z \geq 0.4$. Since $\left\langle\dot{V} / V_{m}\right\rangle$ for the objects with $z \geq 0.4$ is 0.75 (cf. columns 5, 6, 7 of this table) we have chosen $\alpha=2$ (according to equation 18). The exponential evolution laws (equation 20 ) will give numbers for $1 / \mathrm{V}_{\mathrm{m}}^{*}$ very similar to the ones in column 11 (cf. our discussion in section III).

The cumulative luminosity functions obtained from columns. 10 and 11 are plotted on Figure 6. The important features of the luminosity function is the steep rise at high luminosities in contradiction with the flat optical luminosity fraction of equation (31) and a rapid increase at $L \sim 10^{22}$. The latter increase could be evidence for the presence of the class I quasars discussed earlier. If so, we could then calculate the ratio $\left(\rho_{I} / \rho_{I I}\right)$ in equation $(20)$. From Figure $(6)$ we find $\rho_{I} / \rho_{I I} \sim 10^{2}$. Note that at a redshift $z \sim 2, \rho_{I} /\left[\rho_{I I} e^{\beta \tau(z)}\right]<1$ which may indicate that all of the class I quasars were active class II objects at earlier epochs. 
VII. Discussion

We have assumed the redshift of quasars to be cosmological and constructed simple laws for the luminosity function and the statistical evolution of these objects. We have also assumed density evolution. The models developed have come about from the consideration of the difference between optically selected quasars (i.e. quasars of all radio luminosities) and quasars found in radio surveys which have relatively higher radio luminosities. These differences are:

(1) Dependence of $\left\langle\mathrm{v} / \mathrm{v}_{\mathrm{m}}\right\rangle$ on luminosity.

(2) Steeper optical luminosity function for optically selected quasars.

(3) Difference between the redshift distributions, in particular at low redshifts.

(4) Smaller radio to optical luminosity ratio for optically selected objects.

Clearly there exist many possible models which could account for these differences. Schmidt (1970) has suggested that the radio and optical 1uminosities are correlated (as in equation 34 ). This explains the difference in item (2) [and possibly item (4) if the reported (Schmidt 1972) detection of radio signals from some of the optically selected quasars is confirmed] but cannot account for the other differences. Another possibility is modification of the luminosity function of equation (31) such that the optical luminosity function becomes steeper at low values of $F$ (see equation 40 ). This accounts for the differences in items (2) and (4). However, the existence of a relatively large number of low redshift objects among optically selected quasars and the difference between the dependence of $\left\langle\mathrm{V} / \mathrm{v}_{\mathrm{m}}\right\rangle$ on luminosity (if future data show this difference to a more significant level than possible at the present time) imply the need for additional modification of the luminosity function.

One possibility suggested here and earlier (Petrosian 1972) is that 
these differences are due to the presence of two classes (I and II) of quasars. (Note that this in turn implies that the assumption of pure density evolution is incorrect.) The class I quasar typically of $\mathrm{L} \sim 10^{22} \mathrm{w} / \mathrm{Hz}$ are more numerous at the present epoch (by a factor of 100) than the class I I quasars of typically $L \sim 10^{23}$ to $10^{24}$ (Section VI). The class II objects are strong radio emitters $F \sim 10^{26}$ to $10^{28} \mathrm{w} / \mathrm{Hz}$ and show strong density evolution. These are the objects found in radio surveys. The class I objects on the other hand are weak radio emitters $f \lesssim 10^{25.8} \mathrm{w} / \mathrm{Hz}$ and show little or no evolution. These are the low redshift objects found in optically selected samples.

According to some definitions of quasars the class I objects may not be classified as quasars. They may be bright Seyfert or N-type galaxies. They may be objects unrelated to quasistellar radio sources (class II quasars). If, on the other hand the spectroscopic and photometric similarities between the two classes are considered as evidence for some kind of relation between the two classes, then the data discussed here suggests the following picture. The quasars (class I) in their quiescent state are low luminosity $F=F_{Q} \lesssim 10^{25.8} \mathrm{w} / \mathrm{Hz}, \quad L=\mathrm{L}_{Q} \sim 10^{22} \mathrm{w} / \mathrm{Hz}$ nonthermal sources. Occasionally there occur explosions in each source with up to a hundred fold increase in its optical and even larger increase in its radio emission. The result is a class II object. If during these explosions

$$
\mathbf{L}(\mathbf{t})=\mathrm{L}_{\mathbf{Q}}+\frac{\mathrm{F}(\mathrm{t})}{\mathrm{R}}, \frac{\mathrm{F}_{\mathbf{Q}}}{\mathrm{L}_{\mathbf{Q}}}<\mathrm{R}
$$

then the statistical correlation between $L$ and $F$ for radio active quasars can be explained. Furthermore, if the rate and/or duration of such explosions are epoch dependent, then we expect $\left\langle\mathrm{v} / \mathrm{v}_{\mathrm{m}}\right\rangle \neq 0.5$ for class II objects and $\left\langle\mathrm{V} / \mathrm{v}_{\mathrm{m}}\right\rangle \sim 0.5$ for class I objects. (In fact $\left\langle\mathrm{V} / \mathrm{v}_{\mathrm{m}}\right\rangle<0.5$ if these objects could be observed out to a large redshift.) This implies that 
the ratio of the local densities of the two classes $\rho_{I} / \rho_{I I} \geq 300$ to $10^{3}$ depending on the density evolution law of class II sources. The observed value of this ratio is about $10^{2}$. (See Figure 6 and our discussion at the end of Section VI.) This value is obtained from extrapolation of densities of Class II objects from redshifts of order unity to zero, so that this discrepancy does not rule out the model.

If the model is correct than the slope of magnitude counts will be $<0.6$ and $\sim 0.75$ for class I and class II objects, respectively. Since the two classes contribute to the counts approximately equally at 18 magnitude, there should be a change in the slope of the optical counts around this magnitude. Comparison of source counts below 19 magnitude (Sandage, 1965) with the counts above this magnitude (Braccesi and Formiggini, 1969; Sandage and Luyten, 1969) indicate the possibility of an increase in the slope at higher magnitudes. The presence of such a break in the magnitude counts will constitute additional evidence for the model proposed here. of course, data on optically selected samples with larger number of objects and on samples limited to lower radio flux densities $\left(\mathrm{S}_{\mathrm{o}}(178 \mathrm{MHz})<1\right)$ will be useful in testing the model. Further radio observations of optically selected quasars, and in particular limits on the radio flux density of the objects in Schmidt's (1970) sample will also be helpful.

\section{ACKNOWLEDGMENTS}

I would like to thank R. Lynds, M. Schmidt and D. Wills for interesting discussions, and R. Lynds for providing the unpublished data on the optically selected sample. This work was supported by the National Aeronautics and Space Administration under Grant NGR 05-020-510 and the National Science Foundation under Grant GP-23840. 
Bahcal1, J.N., 1972, Ap. J. 172, 265.

Braccesi, A., 1972, in External Galaxies and Quasi-Stellar Objects (Ed. D.E. Evans), p. 453.

Braccesi, A. and Formiggini, L., 1969, Astron. and Astrophys. 3, 364 .

Braccesi, A., Formiggini, L: and Gandolfi, E., 1970, Astron. and Astrophys. 5, 264 .

DeVeney, J.B., Osborn, W.H. and Janes, K., 1971, Pub. Ast. Sco. Pac. 83, 611.

Doroshkevich, A.G., Longair, M.S. and Zeldovic, Ya.B., 1970, Mon. Not. R. Astr. Soc. 147, 139 .

Kafka, P., 1967, Nature 213, 346 .

Lynden-Bel1, B., 1971, Mon. Not. R. astr. Soc. 155, 95.

Lynds, R., Grueff, G. and Braccesi, A., 1972, private communication.

Lynds, R. and Petrosian, V., 1972, Ap. J . 175, 591 .

Lynds, R. and Wills, D., 1972, Ap. J. 172, 531 .

Oke, J.B., Neugebauer, G. and Bek1in, E.E., 1970, Ap. J. 159, 341 .

Petrosian, V., 1970, Astrophys. Letters 6, 71 .

Petrosian, V., 1972, in External Galaxies and Quasi-Stellar Objects (Ed.

D.E. Evans), p. 464 .

Rees, M.J. and Setti, G., 1968, Nature 219, 127.

Rowen-Robinson, M., 1967, Nature 216, 1289.

Sandage, A.R., 1965, Ap. J. 141, 1560 .

Sandage, A.R. and Luyten, W.J., 1969, Ap. J. 155, 913.

Schmidt, M., 1968, Ap. J. 151, 393.

, 1970, Ap. J. 162, 371 .

, 1972, Ap. J. 176, 273.

Wardle, J. F. L., and Miley, G. K., 1971, Ap. J. (Letters), 164, L119. 


\begin{tabular}{|c|c|c|c|c|}
\hline \multirow{7}{*}{ 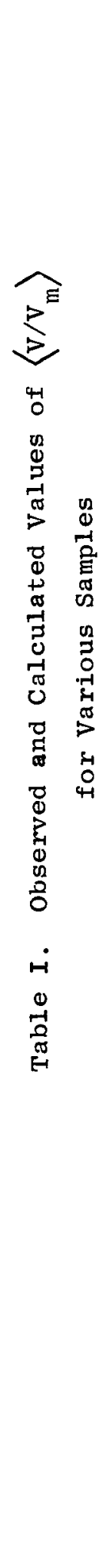 } & \multirow{3}{*}{$\widehat{z}$} & 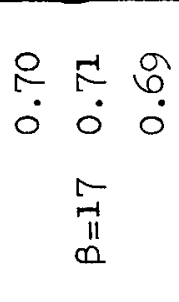 & $\begin{array}{lll}6 & 5 & 6 \\
0 & 0 & 0 \\
& 0 & \\
& 0 & \\
& 11 & \\
0 & & \end{array}$ & $\begin{array}{ccc}\tilde{b} & e & \vec{b} \\
\dot{0} & 0 & 0 \\
& & \\
& n & \\
& n & \\
& 0 & \end{array}$ \\
\hline & & 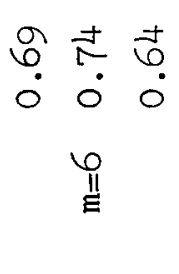 & \begin{tabular}{ccc}
\multirow{0}{*}{} & $N$ & 0 \\
$\dot{0}$ & 0 & 0 \\
& $\overrightarrow{0}$ & \\
& $\vec{E}$ &
\end{tabular} & 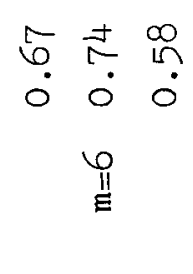 \\
\hline & & $\begin{array}{lll}R & R & R \\
\dot{0} & 0 & 0\end{array}$ & $\begin{array}{lll}\ddots & \ddots & \ddots \\
0 & \ddots & 0 \\
0 & 0 & 0\end{array}$ & $\begin{array}{lll}\vartheta & 0 & n \\
\ddots & \ddots & n \\
0 & 0 & 0\end{array}$ \\
\hline & 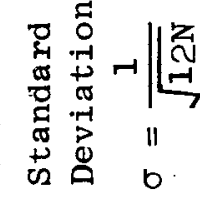 & $\begin{array}{lll}0 & 0 & N \\
1 & 0 & 0 \\
0 & 0 & 0 \\
0 & 0 & 0\end{array}$ & $\begin{array}{lll}m & n & n \\
\stackrel{n}{0} & 5 & 0 \\
0 & 0 & 0 \\
0 & 0 & 0\end{array}$ & $\begin{array}{lll}\overrightarrow{0} & \infty & 0 \\
0 & 0 & 0 \\
0 & 0 & 0\end{array}$ \\
\hline & 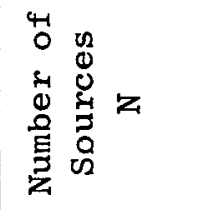 & 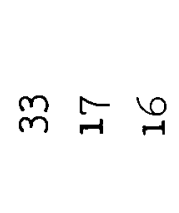 & $\stackrel{m}{n} \stackrel{n}{\rightarrow}$ & 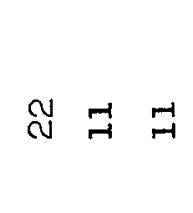 \\
\hline & 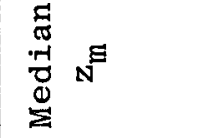 & $\begin{array}{c}0 \\
-1 \\
-1\end{array}$ & $\stackrel{m}{m}$ & $\stackrel{\varphi}{0}$ \\
\hline & $\begin{array}{l}0 \\
\stackrel{0}{1} \\
\stackrel{2}{\tilde{E}} \\
02 \\
02\end{array}$ & 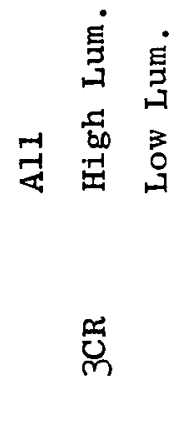 & 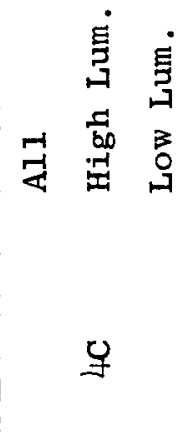 & 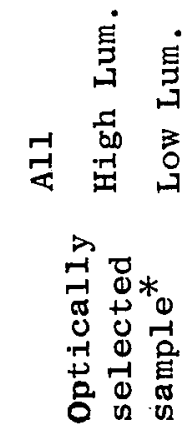 \\
\hline
\end{tabular}

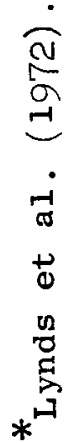




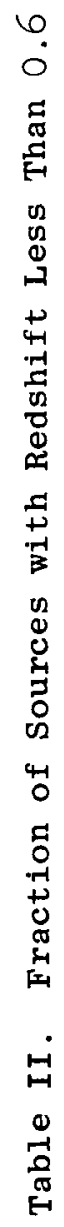

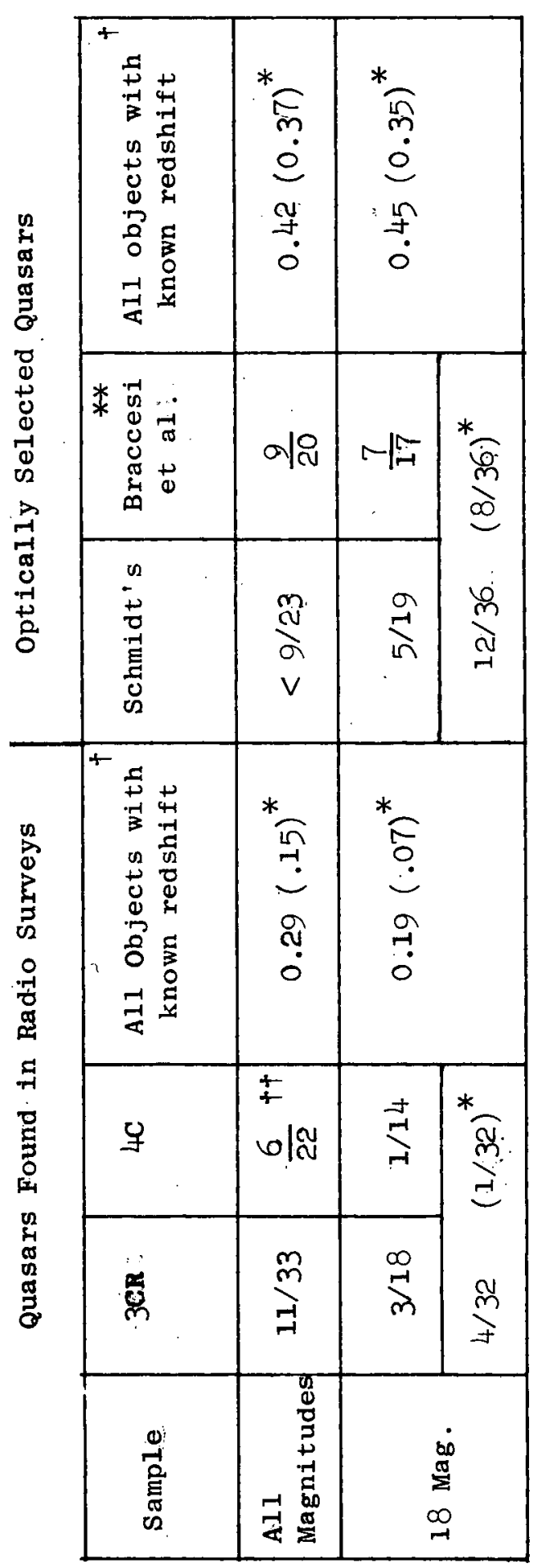

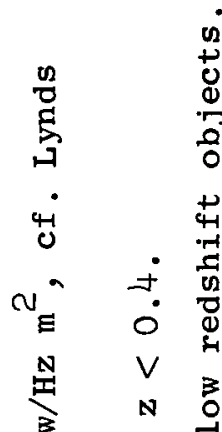

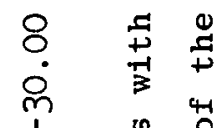

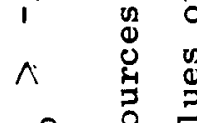

$\sim$ 证

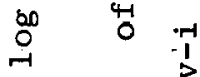

궁

$n$
$\quad \begin{aligned} & 0 \\ & 0\end{aligned}$
0

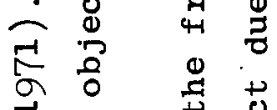

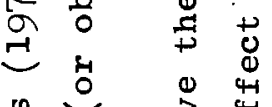

告

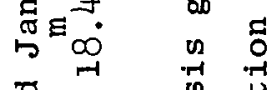

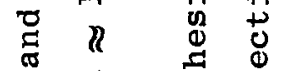

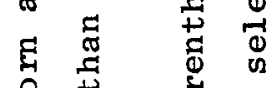

๖ั ฝั

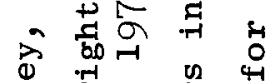

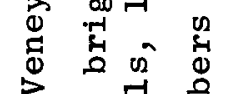

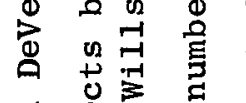

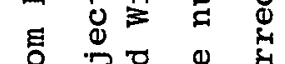

章 


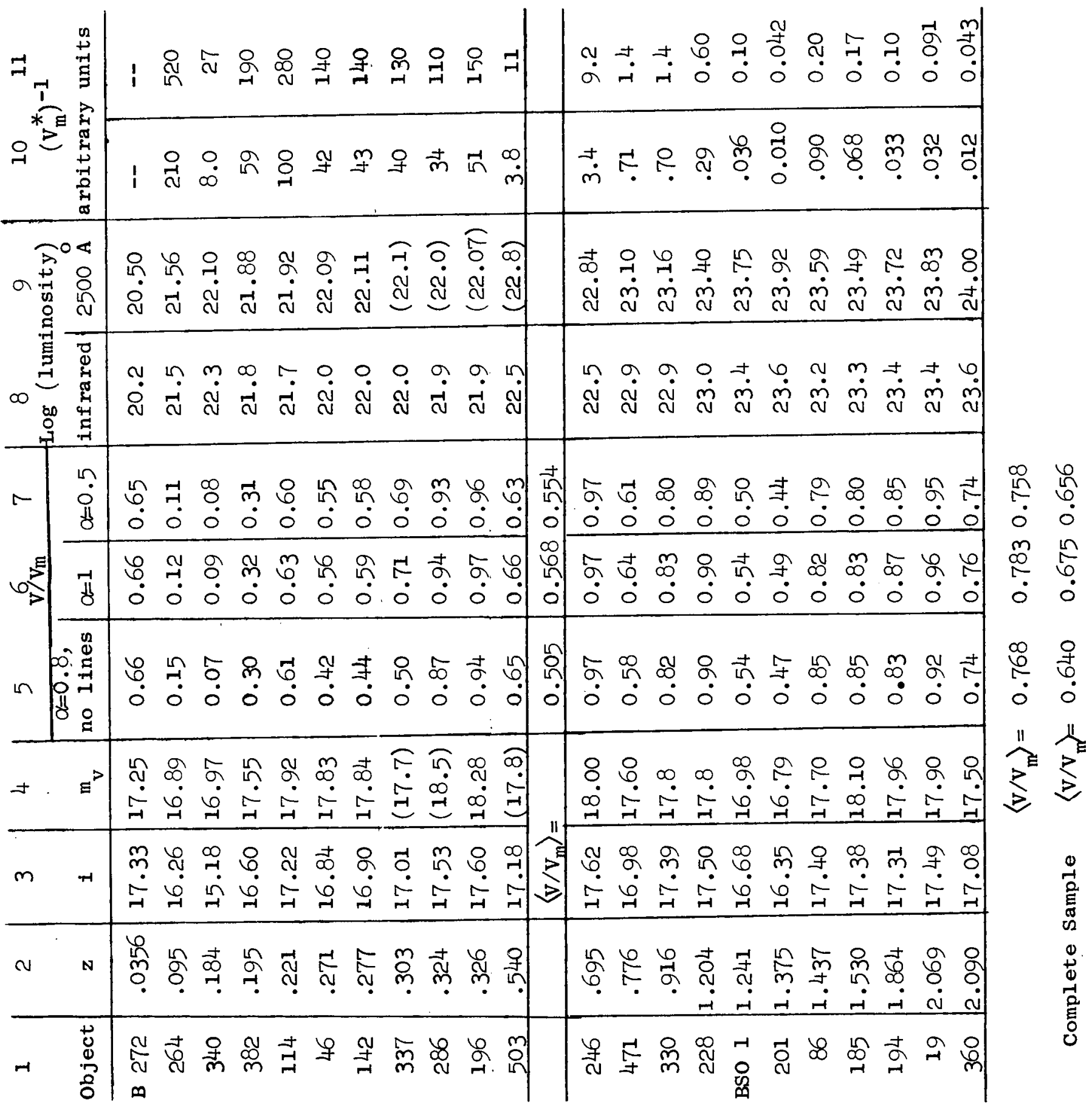




\section{Appendix I}

$\mathrm{V} / \mathrm{V}_{\mathrm{m}}$ Analysis for Samples with Two Limiting Fluxes

In the case of two limiting fluxes the relations between the $\left\langle\mathrm{v} / \mathrm{v}_{\mathrm{m}}\right\rangle$, and the general form of luminosity function $\Psi(L, F, V)$ (with volume $V(z)$ instead of $z$ as epoch indicator) is more complicated. For a given radio and optical limit $S_{0}$ and $I_{0}$, the sources can be divided into two groups. Those with $\frac{F}{\mathrm{~L}}>\frac{\mathrm{S}_{\mathrm{O}}}{\mathrm{I}_{\mathrm{o}}} \equiv \mathrm{R}_{\mathrm{o}}$ or the optically limited objects, and the radio limited objects with $\frac{F}{\mathrm{~L}}<\frac{\mathrm{S}_{\mathrm{O}}}{\mathrm{I}_{\mathrm{o}}}$. Each group then has a single limit and can be treated as described in the text. The total number of sources in the sample is

$$
\mathrm{N}=\mathrm{N}_{\text {opt }}+\mathrm{N}_{\mathrm{rad}}
$$

where the subscripts refer to the limiting flux.

$$
\begin{aligned}
& \mathrm{N}_{\mathrm{opt}}=\int_{0}^{\infty} \mathrm{N}_{\mathrm{opt}}(\mathrm{L}) \mathrm{dL}=\int_{0}^{\infty} \mathrm{dF} \int_{0}^{F / \mathrm{R}_{\mathrm{o}}} \mathrm{dL} \int_{\mathrm{m}}^{\mathrm{V}_{\mathrm{m}}\left(\mathrm{L} / \mathrm{I}_{\mathrm{o}}\right)}{ }_{\Psi(\mathrm{L}, \mathrm{F}, \mathrm{V}) \mathrm{dV},} \\
& \mathrm{N}_{\mathrm{rad}}=\int_{0}^{\infty} \mathrm{N}_{\mathrm{rad}}(\mathrm{L}) \mathrm{dL}=\int_{0}^{\infty} \mathrm{dL} \int_{0}^{\mathrm{LR}} \mathrm{dF} \int_{\mathrm{O}}^{\mathrm{V}_{\mathrm{m}}\left(\mathrm{F} / \mathrm{S}_{\mathrm{O}}\right)}{ }_{\Psi(\mathrm{L}, \mathrm{F}, \mathrm{V}) \mathrm{dV},}
\end{aligned}
$$

where $V_{m}\left(L / I_{o}\right)$ (or $V_{m}\left(F / s_{o}\right)$ are the maximum values for an optically (or radio) limited object with optical (or radio) luminosity L (or F).

The average value of $\mathrm{V} / \mathrm{V}_{\mathrm{m}}$ is therefore given by

$$
\begin{aligned}
& \left\langle\frac{V}{v_{m}}\right\rangle=\frac{1}{N} \int_{0}^{\infty} d L\left\{\int_{0}^{L R} 0 \frac{d F}{v_{m}\left(\frac{F}{S_{o}}\right)} \int_{0}^{V_{m}\left(F / s_{o}\right)} \Psi(L, F, V) V d V\right. \\
& \left.+\int_{L_{0}}^{\infty} \frac{d F}{v_{m}\left(\frac{L}{I_{0}}\right)} \int_{0}^{V_{m}\left(L / I_{0}\right)} \Psi(L, F, V) V d V\right\} .
\end{aligned}
$$

For pure density evolution

$$
\Psi(L, F, V)=\rho_{1}(V) \Psi(L, F)=\rho_{1}(V) \frac{N(L, F)}{\int_{0}^{V} \rho_{1}(V) d V}, \quad V_{m}=V_{m}\left(L / I_{0}\right), \quad F / L>R_{0} .
$$


so that, equation (A.4) is modified as

$$
\left\langle V / v_{m}\right\rangle=\frac{1}{N} \int_{0}^{\infty} d L\left[\int_{0}^{L R} N(L, F) x\left[v_{m}\left(\frac{F}{S_{o}}\right)\right] d F+x\left[v_{m}\left(\frac{L}{I_{0}}\right)\right] \int_{L R_{0}}^{\infty} N(L, F) d F\right],
$$

where

$$
x\left(v_{m}\right)=\frac{\int_{0}^{v_{m}} v_{p_{1}}(v) d v}{v_{m} \int_{0}^{v_{m}} \frac{\rho}{1}(v) d v}
$$

as in equation (13). For discrete sources equation (a6) reduces to

$$
\left\langle v / v_{m}\right\rangle=\frac{1}{N} \sum_{i=1}^{N} x\left(v_{m i}\right)
$$

as in equation (12).

If $x\left(v_{m}\right)$ is independent of $v_{m}\left(d x\left(v_{m}\right) / d v_{m}=0\right)$ equation (A7) and (13) imply

$$
\rho_{1}(v) \propto v^{\alpha}, \quad\left\langle v / v_{m}\right\rangle=\frac{\alpha+1}{\alpha+2}
$$

as in equation (18). In section IV we have refered to variation of $\left\langle\mathrm{V} / \mathrm{v}_{\mathrm{m}}\right\rangle$ with luminosity. For objects of given optical luminosity

$$
\left\langle\frac{V}{V_{m}}\right\rangle_{L}=\left[\int_{0}^{L R_{0}} N\left(I^{\prime}, F\right) x\left[v_{m}\left(\frac{F}{S_{o}}\right)\right] d F+x\left[V_{m}\left(\frac{L^{\prime}}{I_{0}}\right)\right] \int_{L_{0}}^{\infty} N\left(L^{\prime}, F\right) d F\right] / \int_{0}^{\infty} N\left(L^{\prime}, F\right) d F .
$$

In general, independence of this quantity from $L$ does not imply density evolution law of equation (A9). However, if equation (A8) is valid, then for optically limited objects the value of $\left\langle V / V_{m}\right\rangle_{L}=x\left[V_{m}\left(L_{L} / I_{o}\right)\right]$ becomes independent of L. Clearly because of the complete symmetry between $L$ and $F$ the same applies to radio limited objects. 


\section{F IGURE CAPTIONS}

Figure 1. Comparison of various density evolution laws. For each law the exponent is chosen such that $\rho(z=3)=10$.

Figure 2. Variation of expected value of $\mathrm{V} / \mathrm{V}_{\mathrm{m}}$ with the maximum redshift $\mathrm{z}_{\mathrm{m}}$ (or luminosity) for various density evolution laws.

Figure 3. The cumulative luminosity function $\Phi(F, L)$ from the table of Lynds and Wills (1972). (a) The radio luminosity function; the symbols $+, 0, \mathrm{x}$ and stand for $\log \mathrm{L}=22.6,23.0,23.4$ and 22.8, respectively. (b) The optical luminosity function.

Figure 4. Same as in Figure (3), except for $\Phi^{\prime}(R, L), R=F / L$.

Figure 5. Schematic redshift distributions for samples limited to various radio flux density $s_{o}$ (a) Expected from the luminosity function of equation (31). (b) Expected from the luminosity function of equation $(34) . z_{o p t}=\left(\mathrm{L}_{\min } / \mathrm{I}_{\mathrm{o}}\right)^{1 / 2} ; z_{r}=\left(\mathrm{F}_{\mathrm{o}} / \mathrm{S}_{\mathrm{o}}\right)^{1 / 2}$; $z_{r}=\left(R_{\min } L_{\min } / s_{0}\right)^{1 / 2}$.

Figure 6. The cumulative optical luminosity function for optically selected samples of Lynds et al. (1972). Dotts from column 11 and crosses from column 10 of Table III. 


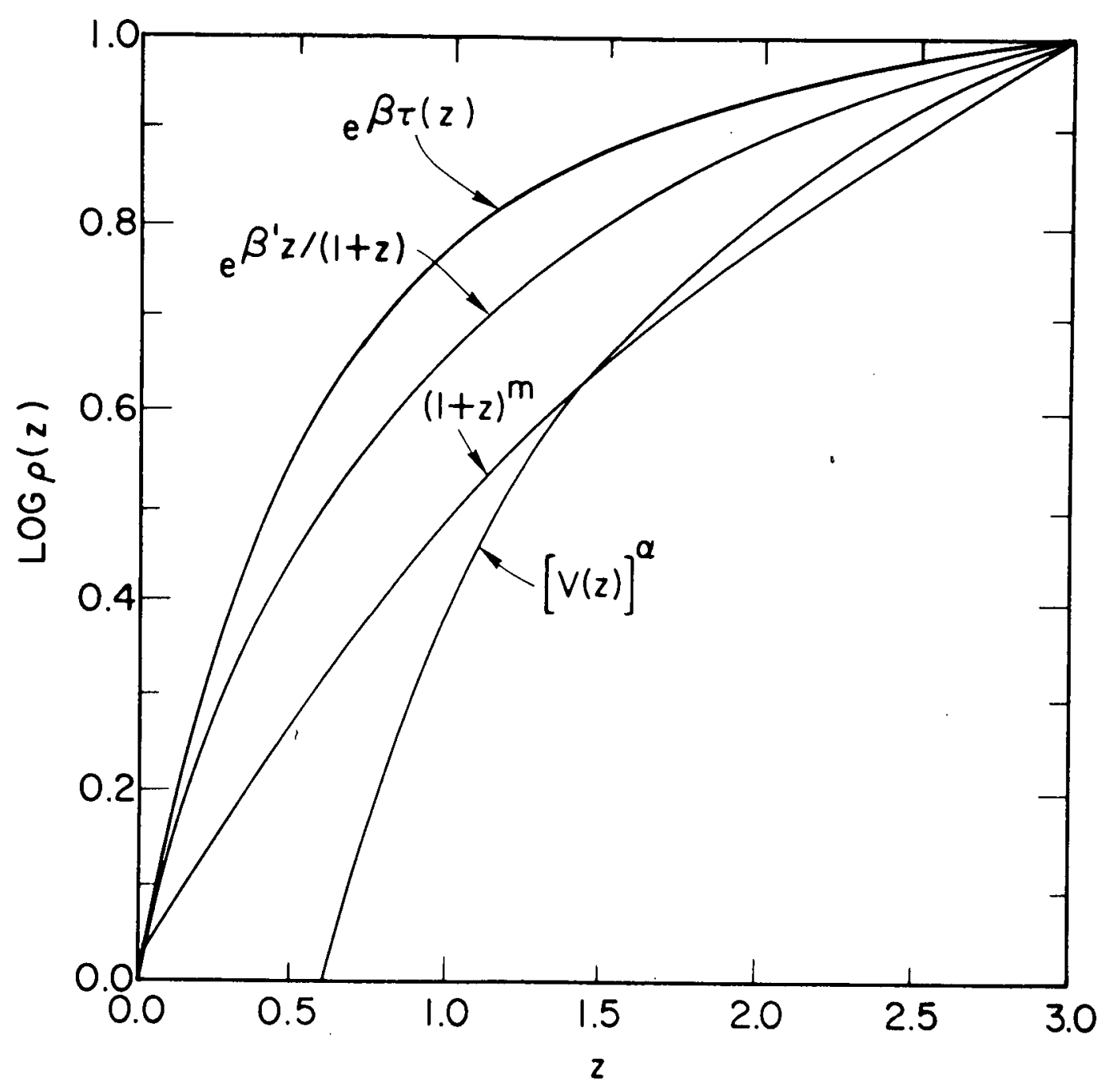

Figure I 


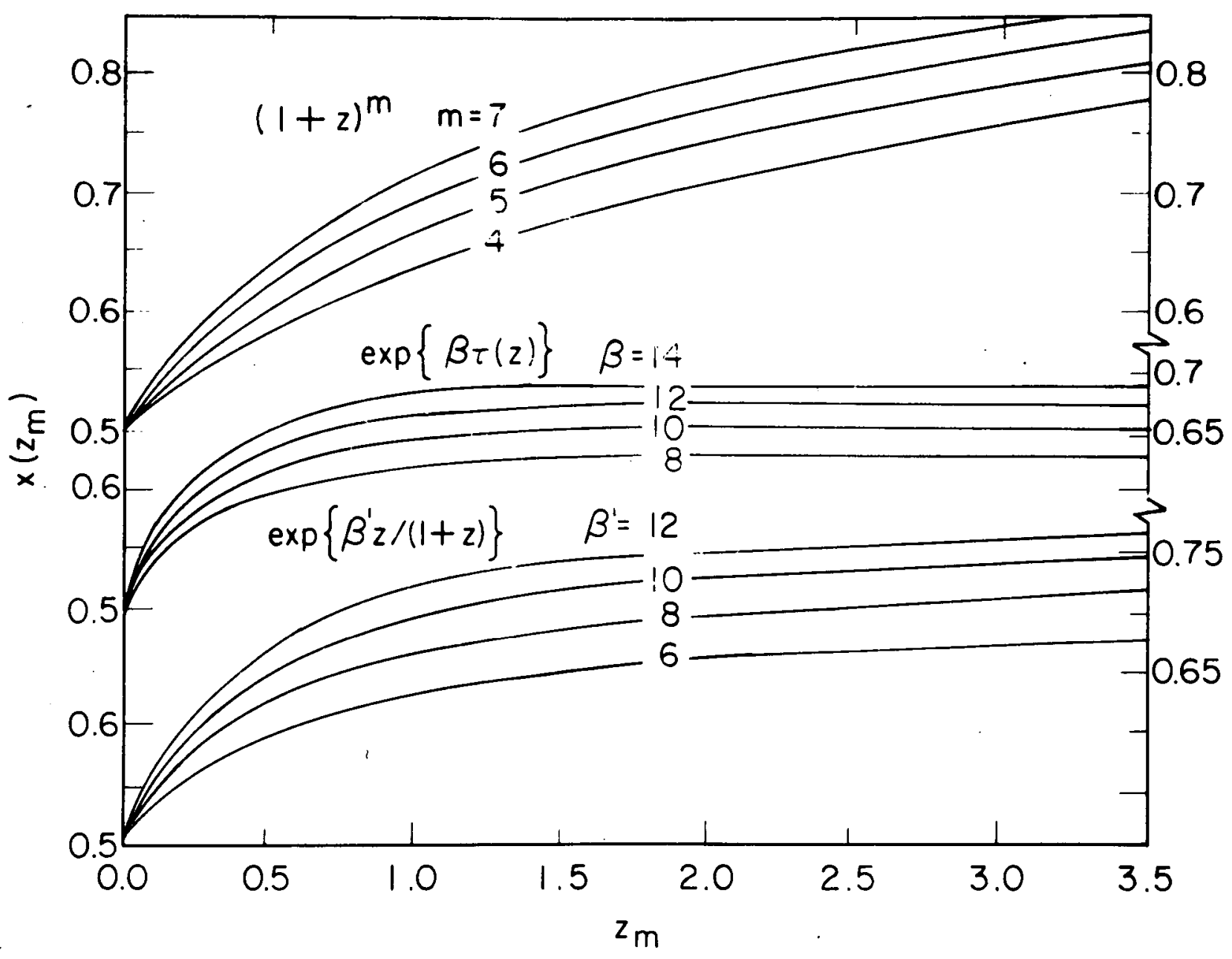

Figure 2 

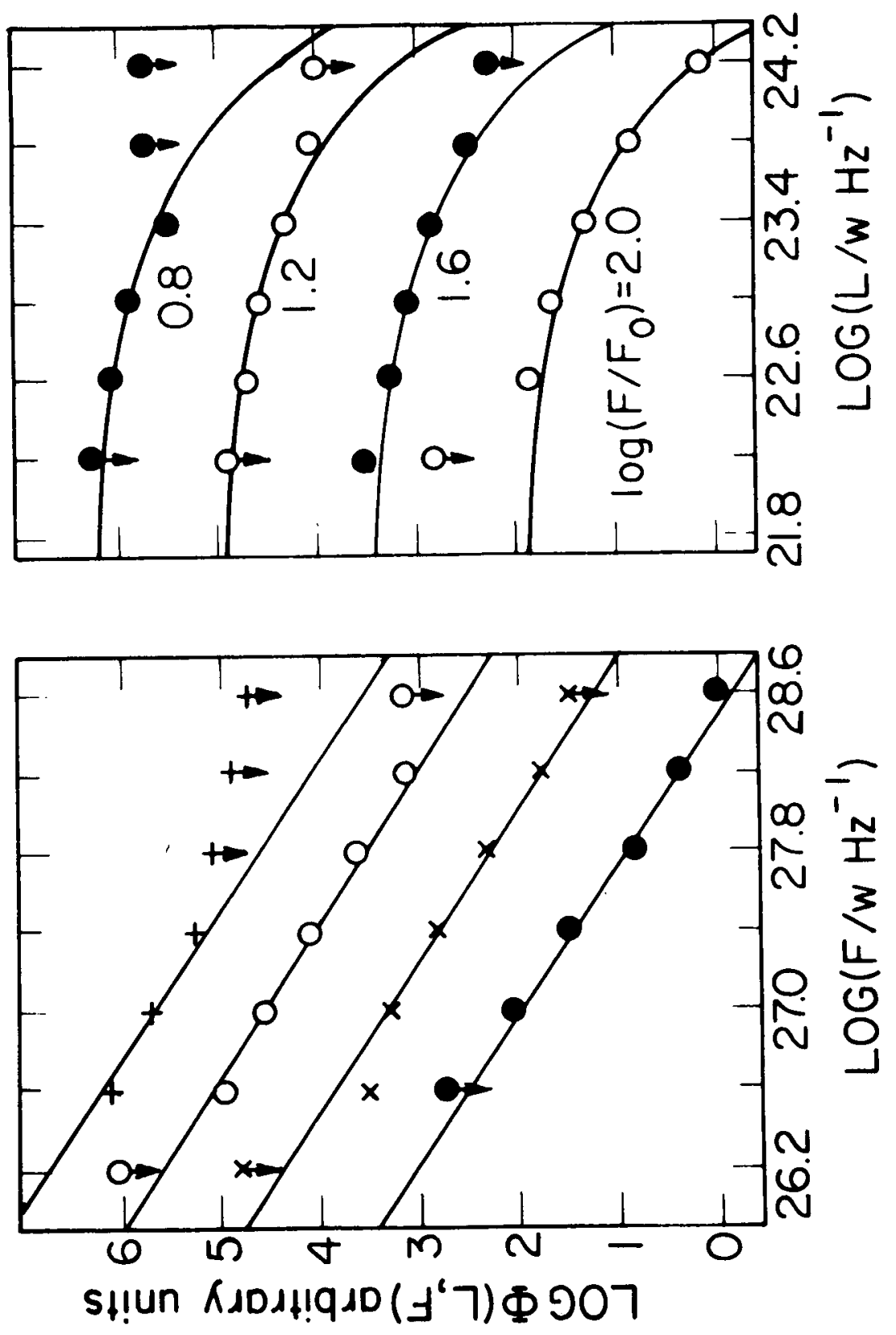

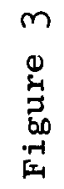



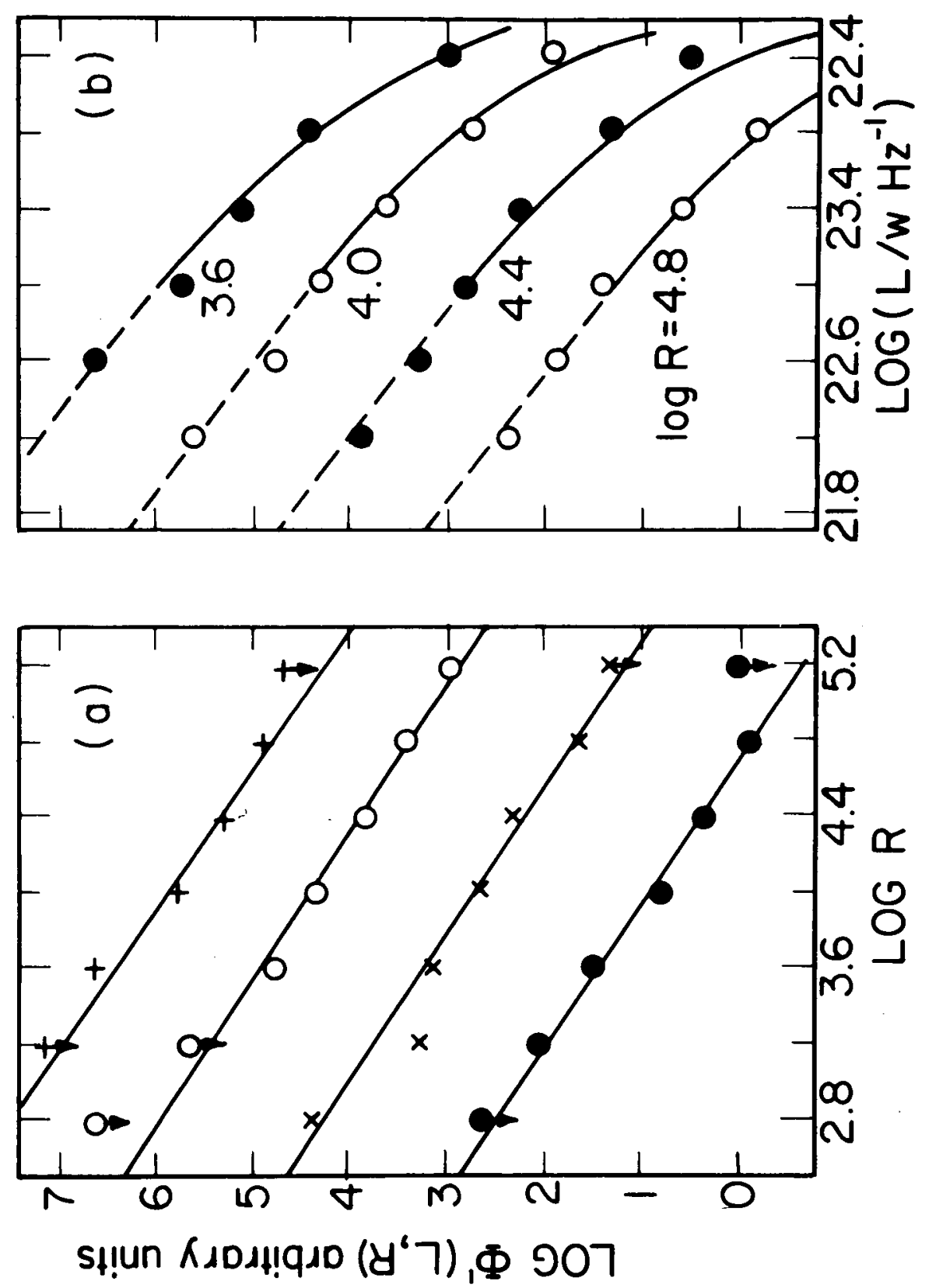

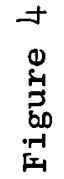




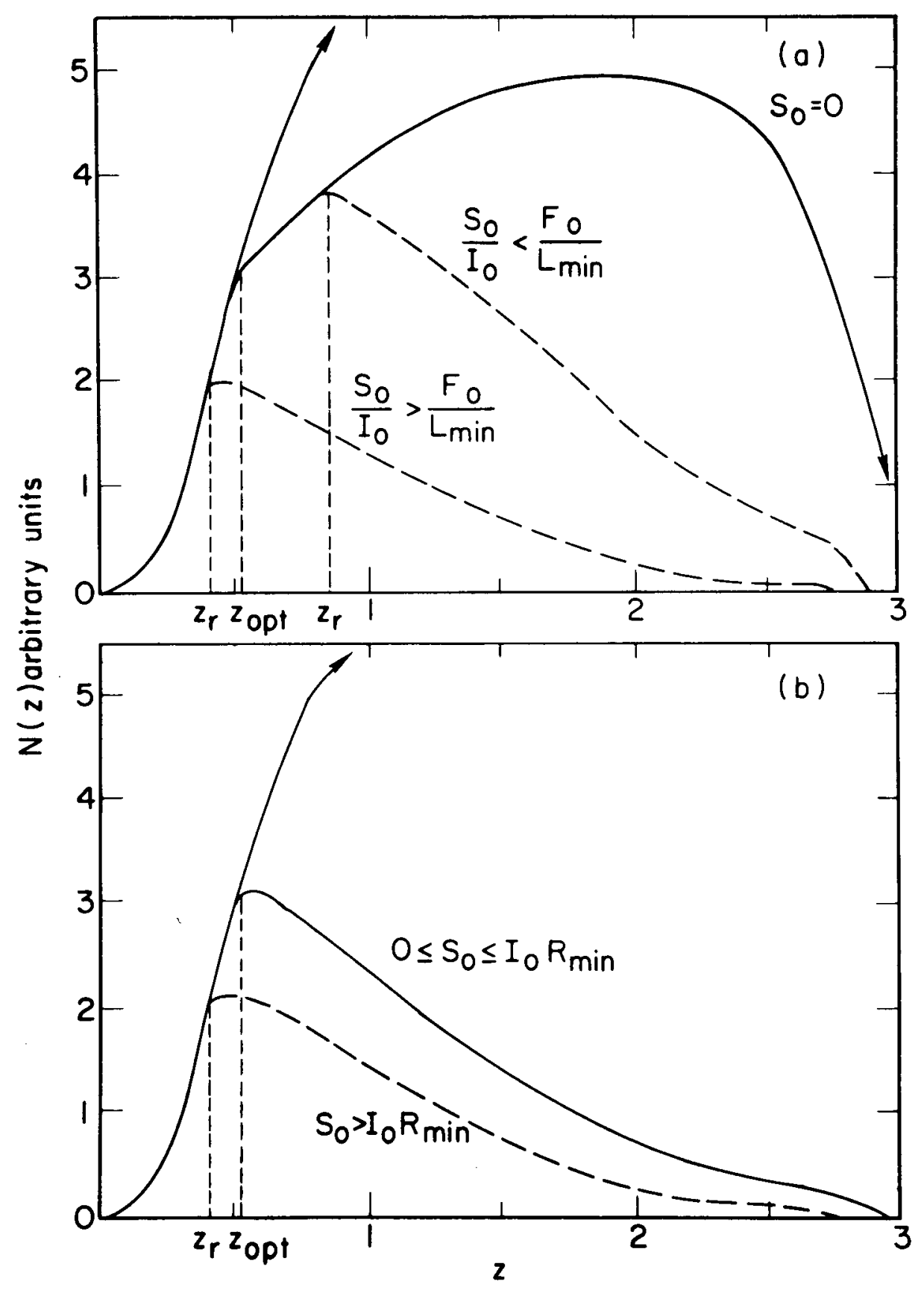

Figure 5 


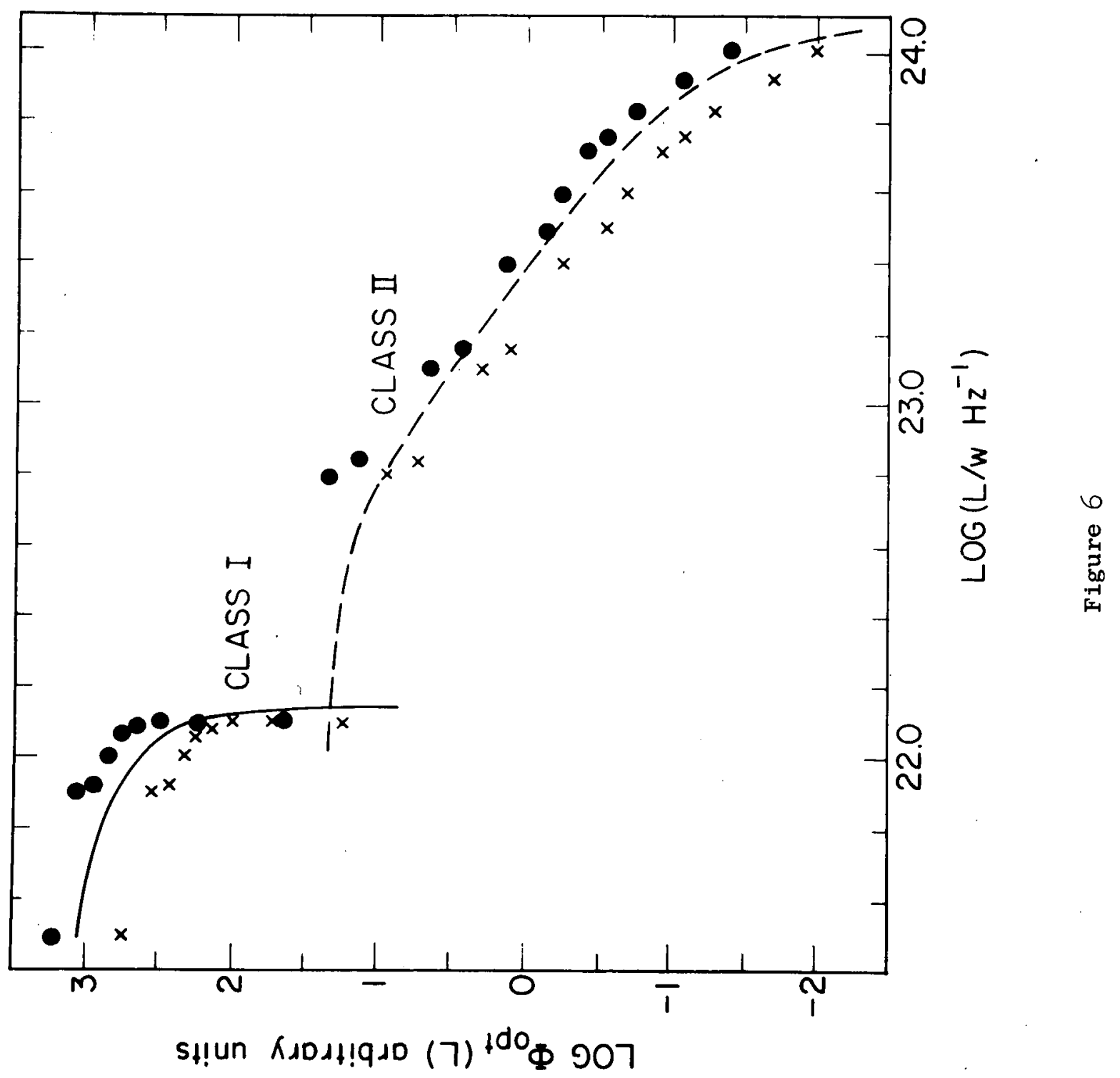

